\title{
Differences between Dorsal Root and Trigeminal Ganglion Nociceptors in Mice Revealed by Translational Profiling
}

\author{
(DSalim Megat, ${ }^{1,2}$ Pradipta R. Ray, ${ }^{1,2}$ Diana Tavares-Ferreira, ${ }^{1}$ Jamie K. Moy, ${ }^{1}$ Ishwarya Sankaranarayanan, ${ }^{1,2}$

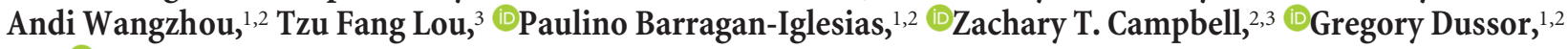 \\ and $\odot$ Theodore J. Price ${ }^{1,2}$ \\ ${ }^{1}$ School of Behavioral and Brain Sciences, ${ }^{2}$ Center for Advanced Pain Studies, and ${ }^{3}$ Department of Biological Sciences, University of Texas at Dallas, \\ Richardson, Texas, 75080
}

\begin{abstract}
Nociceptors located in the trigeminal ganglion (TG) and DRG are the primary sensors of damaging or potentially damaging stimuli for the head and body, respectively, and are key drivers of chronic pain states. While nociceptors in these two tissues show a high degree of functional similarity, there are important differences in their development lineages, their functional connections to the CNS, and recent genome-wide analyses of gene expression suggest that they possess some unique genomic signatures. Here, we used translating ribosome affinity purification to comprehensively characterize and compare mRNA translation in Scn10a-positive nociceptors in the TG and DRG of male and female mice. This unbiased method independently confirms several findings of differences between TG and DRG nociceptors described in the literature but also suggests preferential utilization of key signaling pathways. Most prominently, we provide evidence that translational efficiency in mechanistic target of rapamycin (mTOR)-related genes is higher in the TG compared with DRG, whereas several genes associated with the negative regulator of mTOR, AMP-activated protein kinase, have higher translational efficiency in DRG nociceptors. Using capsaicin as a sensitizing stimulus, we show that behavioral responses are greater in the TG region and this effect is completely reversible with mTOR inhibition. These findings have implications for the relative capacity of these nociceptors to be sensitized upon injury. Together, our data provide a comprehensive, comparative view of transcriptome and translatome activity in TG and DRG nociceptors that enhances our understanding of nociceptor biology.
\end{abstract}

Key words: DRG; mTOR; neuropathic pain; TG; TRAP

Significance Statement

The DRG and trigeminal ganglion (TG) provide sensory information from the body and head, respectively. Nociceptors in these tissues are critical first neurons in the pain pathway. Injury to peripheral neurons in these tissues can cause chronic pain. Interestingly, clinical and preclinical findings support the conclusion that injury to TG neurons is more likely to cause chronic pain and chronic pain in the TG area is more intense and more difficult to treat. We used translating ribosome affinity purification technology to gain new insight into potential differences in the translatomes of DRG and TG neurons. Our findings demonstrate previously unrecognized differences between TG and DRG nociceptors that provide new insight into how injury may differentially drive plasticity states in nociceptors in these two tissues.

\section{Introduction}

Mechanical, thermal, and chemical peripheral stimuli are detected by the pseudo-unipolar sensory neurons of the DRG and

Received 0ct. 15, 2018; revised June 19, 2019; accepted June 20, 2019.

Author contributions:S.M.,P.R.R., Z.T.C., G.D., and T.J.P. designed research;S.M., P.R.R., D.T.-F., J.K.M., I.S., A.W., T.F.L., P.B.-I., and T.J.P. performed research; S.M., P.R.R., and D.T.-F. analyzed data; S.M., P.R.R., A.W., Z.T.C., G.D., and T.J.P. edited the paper; S.M., P.R.R., G.D., and T.J.P. wrote the paper; T.J.P. wrote the first draft of the paper.

This work was supported by National Institutes of Health Grant R01NS065926 to T.J.P., Grant R01NS098826 to T.J.P. and G.D., and Grant R01NS100788 to Z.T.C., University of Texas STARS program to T.J.P. and G.D., and a postdoctoral CONACYT fellowship program to P.B.-I. Raw RNA sequencing data are available through GEO: GSE 113941. Transgenic mice are available through The Jackson Laboratory. All raw data and code are available upon request. the trigeminal ganglion (TG) (Devor, 1999; Woolf and Ma, 2007; Dubin and Patapoutian, 2010). Neurons in the DRG transmit signals from the limbs and body, including much of the viscera, to the CNS through the dorsal horn of the spinal cord. TG neurons relay sensory information from the head and face through a region of the dorsal brainstem known as the trigeminal nucleus caudalis. Although TG and DRG neurons express similar markers and are often considered as very similar, there are differences in

The authors declare no competing financial interests.

Correspondence should be addressed to Theodore J. Price at Theodore.price@utdallas.edu.

https://doi.org/10.1523/JNEUROSCI.2663-18.2019

Copyright $\odot 2019$ the authors 
their cellular populations (Price and Flores, 2007). The tissues also have distinct embryonic origins with important functional consequences (Durham and Garrett, 2010). Finally, neurons in these ganglia innervate distinct targets in the periphery (e.g., the teeth and dura mater for the TG) and in the CNS. An excellent example of this differential innervation in the CNS is the discovery of a subset of TG nociceptors that bypass the traditional second-order relay in the nucleus caudalis projecting directly to the parabrachial nucleus (Rodriguez et al., 2017). These findings suggest distinct molecular signatures of DRG and TG neurons that may be important for understanding sensory neurobiology from these different regions of an organism.

Advances in next-generation sequencing have allowed the characterization of DRG and TG tissues at the genome-wide level using RNA sequencing (RNA-seq) (Manteniotis et al., 2013; Reynders et al., 2015; Gong et al., 2016; Hu et al., 2016; Kogelman et al., 2017). These studies provide significant insight into genes that are differentially expressed between these tissues, including differences between species (Manteniotis et al., 2013; Flegel et al., 2015; Kogelman et al., 2017). However, these studies lack celltype specificity and fail to capture translational efficiency. Cell specificity is a key advantage for single-cell transcriptomic methods (Usoskin et al., 2015; Hu et al., 2016) and other cellular enrichment protocols (Isensee et al., 2014; Thakur et al., 2014; Lopes et al., 2017) that have now been applied to the DRG and/or TG. However, only one direct comparison has thus far been made between TG and DRG transcriptomes using neuronal enrichment followed by RNA-seq (Lopes et al., 2017). Examining ribosome-bound RNA is advantageous because there is strong evidence that transcriptional and translational efficiencies are decoupled in most cells (Fortelny et al., 2017). Methods that sequence ribosome-bound RNAs give more accurate predictions of cellular proteomes (Heiman et al., 2008; Ingolia, 2016). Two techniques have emerged in this area. The first, ribosome footprint profiling, comprehensively and quantitatively provides a snapshot of translation activity at single codon resolution through deep sequencing of ribosome-protected mRNA fragments from cells or tissues (Ingolia, 2016). This technique, which has recently been applied to the DRG (Uttam et al., 2018), does not allow insight into cell-type-specific translational profiling. A second technique is translating ribosome affinity purification (TRAP), which relies on genetic tagging of ribosomal proteins for cell-specific pulldown of translating ribosomes bound to mRNAs for RNA-seq (Doyle et al., 2008; Heiman et al., 2008, 2014). This technique lacks the single codon resolution of ribosome footprint profiling but allows for precise assessment of cellular translatomes in vitro and in vivo.

Here we used the TRAP technology using the Nav1.8 ${ }^{\text {Cre }}$ mouse (Stirling et al., 2005) to achieve sensory neuron-specific ribosome tagging with enrichment in the nociceptor population. We then compared TG and DRG nociceptor translatomes and quantified mRNAs that are differentially expressed at the transcriptional and/or translational level. Interestingly, we found that translational activity of mechanistic target of rapamycin (mTOR)-related genes is higher in the TG compared with DRG. Given the key role that this signaling pathway plays in rapid sensitization of nociceptors (Khoutorsky and Price, 2018), this result is intriguing because activation of nociceptors in the facial region produces greater sensitization and perceived pain in human subjects (Schmidt et al., 2015, 2016), an effect that our experiments also demonstrate in mice. Therefore, our work pinpoints important signaling differences between DRG and TG nociceptors that have direct functional consequences on the susceptibility of these nociceptors to rapid sensitization.

\section{Materials and Methods}

Transgenic animals: Nav1. $8^{\text {cre }} /$ Rosa $26^{\text {fsTRAP }}$ mice. All animal procedures were approved by the Institutional Animal Care and Use Committee of University of Texas at Dallas.

Rosa26 $6^{\text {fSTRAP }}$ mice were purchased from The Jackson Laboratory (stock \#022367). Transgenic mice expressing Cre recombinase under the control of the Scn10a (Nav1.8) promoter were obtained initially from Professor John Wood (University College London) but are commercially available from Infrafrontier (EMMA ID: 04582). The initial characterization of these mice demonstrated that the introduction of the Cre recombinase in heterozygous animals does not affect pain behavior, and their DRG neurons have normal electrophysiological properties (Stirling et al., 2005). Nav1.8 ${ }^{\text {cre }}$ mice on a C57BL/6J genetic background were maintained and bred at the University of Texas at Dallas. Upon arrival, Rosa $26^{\text {fsTRAP }}$ mice were crossed to Nav1.8 ${ }^{\text {cre }}$ to generate the Nav1.8TRAP mice that express a fused EGFP-L10a protein in Nav1.8-expressing neurons. All experiments were performed using male and female littermates 8-12 weeks old. Mice were group housed (4 maximum) in nonenvironmentally enriched cages with food and water ad libitum on a $12 \mathrm{~h}$ light-dark cycle. Room temperature was maintained at $21 \pm 2{ }^{\circ} \mathrm{C}$.

TRAP. Nav1.8-TRAP male and female mice were decapitated and DRG and TG rapidly dissected in ice-cold dissection buffer $(1 \times$ HBSS; Invitrogen, 14065006), $2.5 \mathrm{~mm}$ HEPES, $35 \mathrm{~mm}$ glucose, $4 \mathrm{~mm} \mathrm{NaHCO}$, $100 \mu \mathrm{g} / \mathrm{ml}$ cycloheximide, $0.001 \mathrm{~V} 2 \mathrm{mg} / \mathrm{ml}$ emetine). DRGs or TGs were transferred to ice-cold polysome buffer (20 mM HEPES, 12 mм $\mathrm{MgCl}_{2}$, $150 \mathrm{~mm} \mathrm{KCl}, 0.5 \mathrm{~mm}$ DTT, $100 \mu \mathrm{g} / \mathrm{ml}$ cycloheximide, $20 \mu \mathrm{g} / \mathrm{ml}$ emetine, $40 \mathrm{U} / \mathrm{ml}$ SUPERase IN, Promega, $1 \mu \mathrm{l}$ DNase, and protease inhibitor) and homogenized using a Dounce homogenizer. Samples were centrifuged at $3000 \times g$ for $10 \mathrm{~min}$ to prepare postnuclear fraction (S1). Then, $1 \% \mathrm{NP}-40$ and $30 \mathrm{~mm}$ 1,2-dihexanoyl-sn-glycero-3-phosphocholine were added to the $\mathrm{S} 1$ fraction and then centrifuged at $15,000 \times g$ for 15 min to generate a postmitochondrial fraction (S20). A $200 \mu \mathrm{l}$ sample of S20 was removed for use as Input, and $800 \mu \mathrm{l}$ of S20 was incubated with protein G-coated Dynabeads (Invitrogen) bound to $50 \mu \mathrm{g}$ of anti-GFP antibodies (HtzGFP-19F7 and HtzGFP-19C8, Memorial Sloan Kettering Centre) for $3 \mathrm{~h}$ at $4^{\circ} \mathrm{C}$ with end-over-end mixing. Anti-GFP beads were washed with high salt buffer (20 mM HEPES, $5 \mathrm{~mm} \mathrm{MgCl}_{2}, 350 \mathrm{~mm} \mathrm{KCl}$, $1 \% \mathrm{NP}-40,0.5 \mathrm{~mm}$ DTT, and $100 \mu \mathrm{g} / \mathrm{ml}$ cycloheximide), and RNA was eluted from all samples using a Direct-zol kit (Zymo Research) according to the manufacturer's instructions. RNA yield was quantified using a Nanodrop system (Thermo Fisher Scientific), and RNA quality was determined by fragment analyzer (Advanced Analytical Technologies).

Library generation and sequencing. Libraries were generated from 100 ng to $1 \mu \mathrm{g}$ of total RNA using Quantseq 3' mRNA-Seq library kit (Lexogen) with RiboCop rRNA depletion kit (Lexogen) treatment according to the manufacturer's protocols. The endpoint PCR amplification cycle number for each sample was determined by qPCR assay with PCR Add-on kit for Illumina (Lexogen). The cycle number was selected when the fluorescence value reached $33 \%$ of the maximum for each sample. Purified libraries were quantified by Qubit (Invitrogen), and the average size was determined by fragment analyzer (Advanced Analytical Technologies) with high-sensitivity next-generation sequencing fragment analysis kit. Libraries were then sequenced on an Illumina NextSeq500 Sequencer using $50 \mathrm{bp}$ single-end reads.

Sequence files generated by the Illumina NextSeq500 Sequencer were downloaded from BaseSpace. An initial quality check using FastQC 0.11.5 (Babraham Bioinformatics) was done on the sequencing files, and then trimming was performed on the server with the FASTQ Toolkit. Sequences were trimmed with optimized parameters (13 bases from 3' end, 17 bases from $5^{\prime}$ end, and any poly-adenine longer than 2 bases from the $3^{\prime}$ side). Trimming parameters were optimized based on FastQC results and mapping rate, as well as manually checking high reads or abundant chromosomal regions with IGV 2.3.80. The trimmed sequencing samples were then processed using TopHat 2.1.1 (with Bowtie 2.2.9) and mapped to the mouse reference genome (NCBI reference assembly GRCm38.p4) and reference transcriptome (Gencode vM10) generating 
files in .bam format. Processed .bam files were then quantified for each gene using Cufflinks 2.2.1 with gencode.vM10 genome annotation. Because reads only mapped to the $3^{\prime}$ UTR of the gene, read counts were not normalized by length by using the Cufflinks option - no-lengthcorrection. Relative abundance for the $\mathrm{i}^{\text {th }}$ gene was determined by calculating TPM (transcripts per million) values as follows:

$$
\operatorname{TPM}_{i}=10^{6} \times \frac{a_{i}}{\sum_{j}\left[a_{j}\right]}
$$

where $a_{j}$ is the Cufflinks reported relative abundance. Finally, TPM values were normalized to upper decile for each biological replicate, and udTPM (upper decile TPM) were used for analysis (Glusman et al., 2013). This was done to provide uniform processing for samples with varying sequencing depth and because of varying number of genes in the transcriptome and translatome samples.

Behavioral procedures. Female C57BL/6J mice were injected subdermally with capsaicin $(0.1 \mu \mathrm{M})$ into either cheek or hindpaw in a volume of $10 \mu \mathrm{l}$ with Hamilton syringe and $30 \mathrm{G}$ needle. For cheek injections, mice cheeks were shaved $3 \mathrm{~d}$ before injections. AZD8055 (mTORC1 inhibitor) or vehicle was administered intraperitoneally $(10 \mathrm{mg} / \mathrm{kg}) 2 \mathrm{~h}$ before capsaicin injections into the cheek. AZD8055 was dissolved in DMSO (50 $\mathrm{mg} / \mathrm{ml}$ ) and further diluted in $30 \%(\mathrm{w} / \mathrm{v})$ cyclodextrin to make up the correct dose for each animal. Vehicle consisted of 10\% DMSO and 30\% w/v cyclodextrin. Baseline videos were recorded for 15 min for each mouse. After cheek or hindpaw injections, experimental videos were recorded for $60 \mathrm{~min}$. The recording setup consisted of one camera in front and one in the back. The sum of facially directed behaviors with the forepaws following injection of capsaicin into the whisker pad as well as the number of hindpaw directed behaviors for the hindpaw were scored and classified as nocifensive behaviors.

The Mouse Grimace Scale was used to quantify affective aspects of pain in mice (Langford et al., 2010). We scored the changes in the facial expressions (using the facial action coding system) at baseline and then 15 and $30 \mathrm{~min}$ after intraplantar or facial injection of capsaicin.

$q R T-P C R$. Lumbar DRGs and TGs were isolated from 4 male mice per genotype and flash-frozen on dry ice and stored at $-80^{\circ} \mathrm{C}$ until ready to be processed. Tissues were homogenized using a pestle, and total RNA was extracted using RNAqueous Total RNA Isolation kits (Thermo Fisher Scientific). RNA was subsequently treated with TURBO DNase (Thermo Fisher Scientific) according to the manufacturer's instructions. RNA concentration was measured on a NanoDrop 2000 (Thermo Fisher Scientific). cDNA was synthesized using iScript Reverse Transcriptase (Bio-Rad). qRT-PCR was done using a Applied Biosystems Lightcycler 7500 Real-Time PCR system using iTaq Universal SYBR Green Supermix (Bio-Rad) according to the manufacturer's instructions with 3 technical replicates per biological replicate (averages of the technical replicates per biological replicate are reported) using primers pairs: Gapdh forward 5'-GACAACTTTGGCATTGTGGA-3' and Gapdh reverse 5'-CATCA TACTTGGCAGGTTTCTC-3', Rraga forward 5'-ACGTCCGATTCT TGGGGAAC- $3^{\prime}$ and Rraga reverse $5^{\prime}$-TACGGAAGATGTTGTCCCGC3', Fth forward 5'-GCACTGCACTTGGAAAAGAGT-3' and Fth reverse 5'-ACGTGGTCACCCAGTTCTTT-3'. Primers were made by Integrated DNA Technologies.

Primer efficiency curves were determined by diluting total RNA of DRG and TG samples with 6 points of 1:5 serial dilutions. RNA dilutions were then converted to CDNA, and standard curves were determined for DRG and TG with each primer set separately. Concentrations resulting in multiple products or incorrect product size via melt-curve analysis (derivative reporter vs temperature) were omitted. Efficiencies for each primer set for DRGs and TGs were calculated using the Applied Biosystems 7500 software version 2.3. Total RNA (115 ng) used in experiments fell within primer standard curves with efficiencies between $85 \%$ and $110 \%$. Data were analyzed as $2^{-\Delta \Delta C t}$ and normalized as shown in Results.

Antibodies. The peripherin antibody used for immunohistochemistry were obtained from Sigma-Aldrich. Isolectin $\mathrm{B}_{4}\left(\mathrm{IB}_{4}\right)$ conjugated to AlexaFluor-568 and secondary AlexaFluor antibodies were purchased from Invitrogen. Calcitonin gene-related peptide (CGRP) antibody was purchased from Peninsula Laboratories. RagA and Akt1s1 (also known as PRAS40) antibodies were from Cell Signaling Technology. Antibodies for TRAP (HtzGFP-19F7 and HtzGFP-19C8) were obtained from Sloan Memorial Kettering Centre, after establishing Material Transfer Agreements with the laboratory of Prof. Nathaniel Heintz (Rockefeller University).

Immunohistochemistry. Animals were anesthetized with isoflurane (4\%) and killed by decapitation, and tissues were flash-frozen in OCT on dry ice. Sections of TG $(20 \mu \mathrm{m})$ were mounted onto SuperFrost Plus slides (Thermo Fisher Scientific) and fixed in ice-cold 10\% formalin in $1 \times$ PBS for $45 \mathrm{~min}$, and then subsequently washed 3 times for $5 \mathrm{~min}$ each in $1 \times$ PBS. Slides were then transferred to a solution for permeabilization made of $1 \times$ PBS with $0.2 \%$ Triton X-100 (Sigma-Aldrich). After $30 \mathrm{~min}$, slides were washed 3 times for $5 \mathrm{~min}$ each in $1 \times$ PBS. Tissues were blocked for at least $2 \mathrm{~h}$ in $1 \times \mathrm{PBS}$ and $10 \%$ heat-inactivated normal goat serum. TG or DRG slices were stained with peripherin, CGRP, and $\mathrm{IB}_{4}$ conjugated to AlexaFluor-568. Immunoreactivity was visualized following $1 \mathrm{~h}$ incubation with goat anti-rabbit, goat anti-mouse, and goat anti-guinea pig AlexaFluor antibodies at room temperature. All immunohistochemical images are representations of samples taken from 3 animals per genotype. Images were taken using an Olympus FluoView 1200 confocal microscope. Analysis of images was done using ImageJ Version 1.48 for Apple OSX (National Institutes of Health).

Western blotting. Male and female mice were used for all Western blotting experiments and were killed by decapitation while under anesthesia and tissues (DRG or TG) were flash frozen on dry ice. Frozen tissues were homogenized in lysis buffer ( $50 \mathrm{~mm}$ Tris, pH 7.4, $150 \mathrm{~mm}$ $\mathrm{NaCl}, 1$ mм EDTA, pH 8.0, and 1\% Triton X-100) containing protease and phosphatase inhibitors (Sigma-Aldrich), and homogenized using a pestle. A total of $15 \mu \mathrm{g}$ of protein was boiled for $5 \mathrm{~min}$ in loading dye and then loaded into each well and separated by a 10\%-12\% SDS-PAGE gel. Proteins were transferred to a $0.45 \mu \mathrm{m}$ PVDF membrane (Millipore) at $25 \mathrm{~V}$ overnight at $4^{\circ} \mathrm{C}$. Subsequently, membranes were blocked with $5 \%$ nonfat dry milk in $1 \times$ Tris buffer solution containing Tween 20 (TTBS) for $3 \mathrm{~h}$. Membranes were washed in $1 \times$ TTBS 3 times for 5 min each, then incubated with primary antibody overnight at $4^{\circ} \mathrm{C}$. The following day, membranes were washed 3 times in $1 \times$ TTBS for 5 min each, then incubated with the corresponding secondary antibody at room temperature for $1 \mathrm{~h}$. Membranes were then washed with $1 \times$ TTBS 5 times for $5 \mathrm{~min}$ each. Signals were detected using Immobilon Western Chemiluminescent HRP substrate (Millipore). Bands were visualized using film (Kodak) or with a Bio-Rad ChemiDoc Touch. Membranes were stripped using Restore Western Blot Stripping buffer (Thermo Fisher Scientific) and reprobed with another antibody. Analysis was performed using Image Lab (Bio-Rad).

Statistics. All data are presented as mean \pm SEM. All analysis was done using GraphPad Prism 6 version 6.0 for Mac OS X. Single comparisons were performed using Student's $t$ test or one-way ANOVA if multiple groups were compared. For behavioral experiments, two-way ANOVA (time $\times$ treatment) was used to measure effects across time between different groups. If significant effects were found by ANOVA, post hoc analyses were performed. Multiple comparisons between groups/within groups were performed using Sidak's correction. Statistical results can be found in the figure legends.

Statistics for RNA sequencing. Differential expression analysis was performed using MATLAB scripts. TPM values were normalized to their 90th percentile to generate udTPMs, and the probability density function of the udTPM was used to set the threshold value for further analysis. Genes showing consistent expression above the set threshold across biological replicates were then used to generate lists of differentially expressed genes. Standard $t$ test was first performed assuming unequal variances between experimental groups generating $p$ values for each gene as follows. A $q$ value for the $\mathrm{i}^{\text {th }}$ test was then calculated using BenjaminiHochberg correction for multiple comparisons as follows:

$$
\text { Qvalue }_{i}=\frac{\text { pvalue }_{i} \times N}{\text { rank }_{\text {pualue }_{i}}}
$$

where $N$ is the number of tests. 

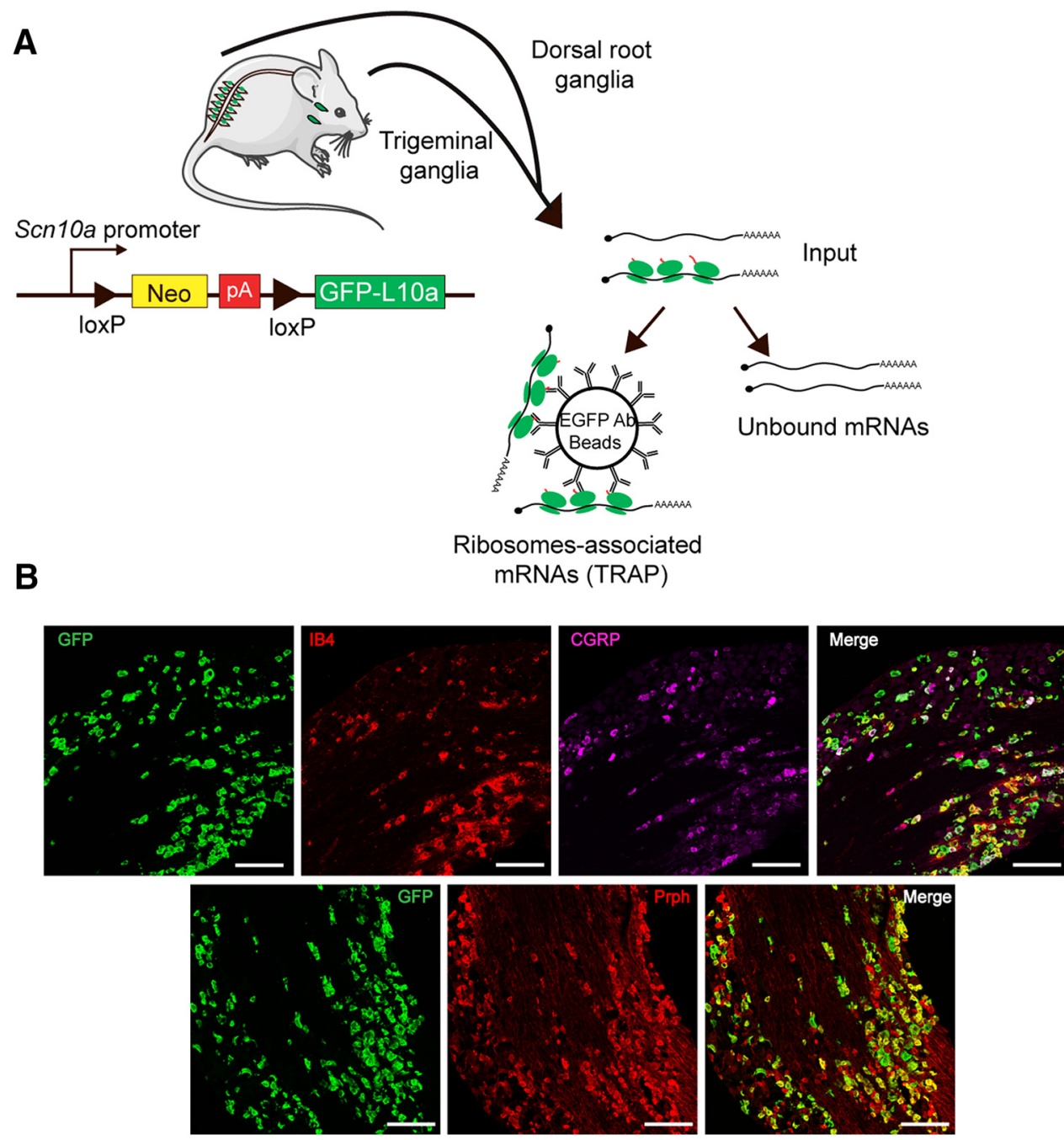

Figure 1. TRAP-seq strategy and expression in TG. A, Schematic representation of TRAP-seq approach showing isolation of translating ribosomes with immunoprecipitation using anti-GFPcoated beads. $\boldsymbol{B}$, Immunostaining of $\mathrm{CGRP}_{\text {, IB }}$, and peripherin (Prph) on TG sections from Nav1.8-TRAP mice (GFP). Scale bar, $100 \mu \mathrm{m}$.

Finally, the cumulative density function of the fold change was plotted and used to set the fold change for the input and TRAP fraction for both DRG and TG datasets. Gene set enrichment analyses were performed with Enrichr (Kuleshov et al., 2016) using the Gene Ontology molecular function 2015 term, the biological process 2015 term, and the Reactome 2015 libraries.

For motif finding, $5^{\prime}$-UTR sequences of corresponding genes were obtained from gencode.vM10 (mouse genome assembly GRCm38), with all transcript isoforms kept for analysis. As most 5'-UTRs of different isoforms from the same gene share partial/whole sequences with each other, when a $5^{\prime}$-UTR sequence was fully shared by another longer $5^{\prime}$ UTR isoform of the same gene, the shorter version was removed to prevent genes with a large amount of isoforms being overrepresented in the motif analysis. All 5'-UTR sequences remaining after filtering were then passed through MEME Suite 5.0.2 for motif discovery, with the following parameters: all motifs are within 10-20 bp length range, only found on the provided strand, and appear in at least $10 \%$ of the genes provided. Motifs appearing in $>30 \%$ of the genes with significant $E$ value are shown in the text.

\section{Results}

To generate nociceptor-TRAP mice, Nav1.8 ${ }^{\text {cre }}$ animals were crossed with Rosa26 ${ }^{\text {fs-TRAP }}$ (Zhou et al., 2013) to express the eGFP fused to the ribosomal L10a protein in Nav1.8 ${ }^{+}$neurons. This approach generates Nav1.8-TRAP neurons in both the
DRG and TG. While the specificity of our approach was recently shown in the DRG (Megat et al., 2019), we characterized expression of the transgene in the TG (Fig. 1A). We found that eGFP-L10a-positive neurons primarily colocalized with smalldiameter peripherin-positive neurons and that extensive overlap was found with both CGRP immunoreactivity and with $\mathrm{IB}_{4}$ staining (Fig. 1B). These findings demonstrate that this technique labels an equivalent subset of neurons in the DRG and TG of mice.

Having confirmed that the Nav1.8-TRAP approach yields robust expression in nociceptors in the TG, we set out to conduct TRAP sequencing to compare nociceptor translatomes in the DRG and TG. To successfully isolate ribosome-associated mRNAs from Nav1.8-TRAP cells, we determined that TGs from 4 animals were required for a single biological replicate. This number matches the number of DRGs needed for TRAP sequencing. To make comparisons between the TG and DRG, we generated TRAP sequencing from the TG that was then compared with our previously generated DRG dataset (GSE 113941). We sequenced the total mRNA input from all biological replicates and mRNAs associated with translating ribosomes in the Nav1.8 subset of TG neurons, equivalently to what was done from DRG (Megat et al., 2019). This approach allowed us to make comparisons between 
A

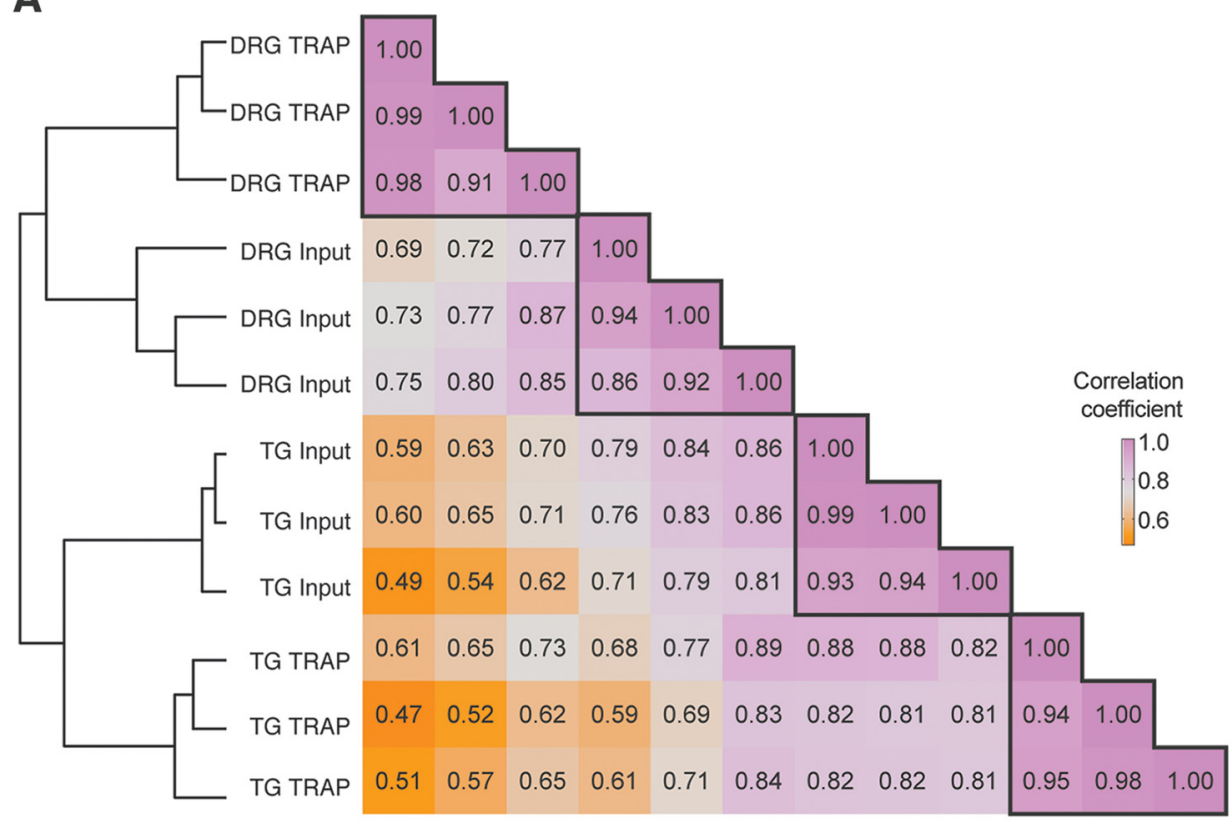

B
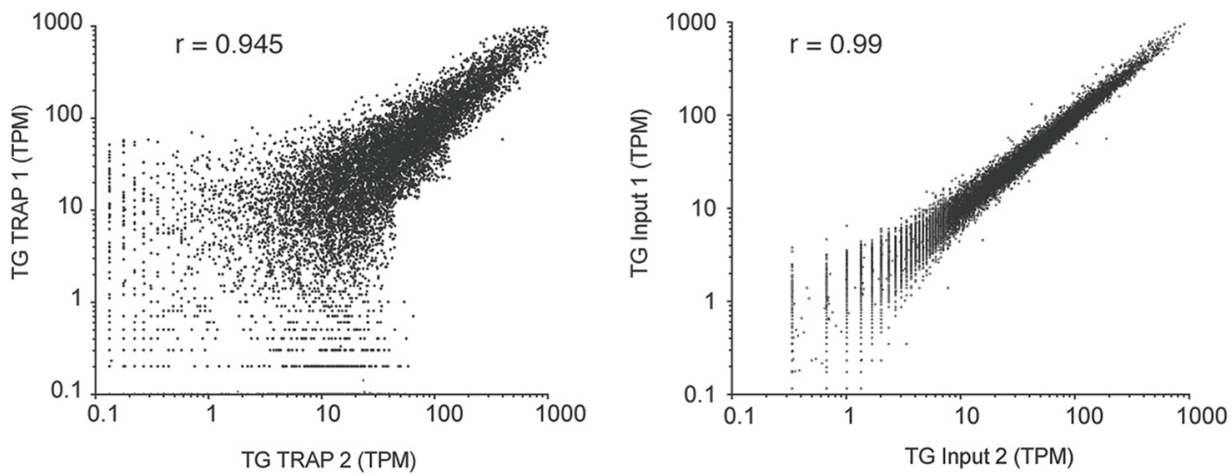

C

Nav1.8 - TRAP
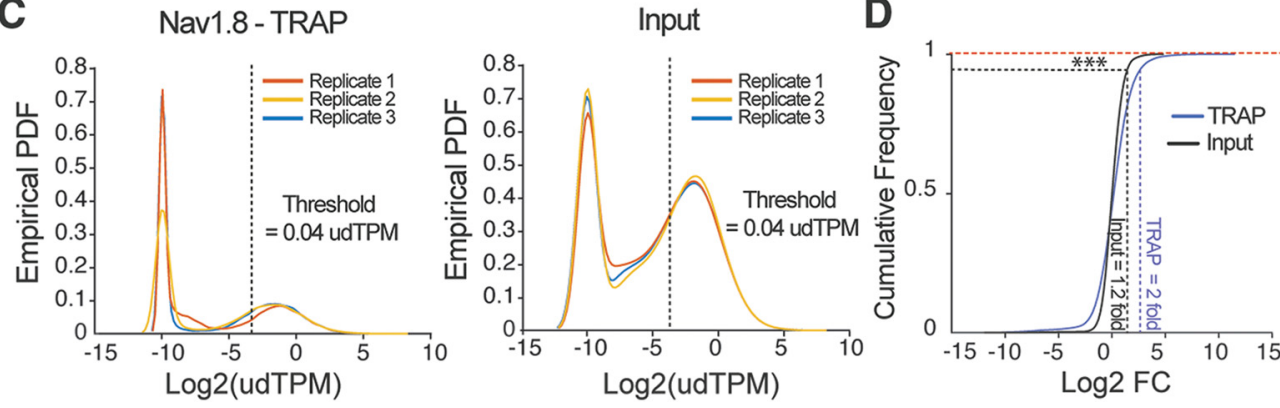

Figure 2. DRG and TG TRAP-seq shows high correlation between biological replicates and similar sequencing depth. $\boldsymbol{A}$, Heatmap of the correlation coefficient and cluster analysis showing clear separation between DRG and TG as well as in between TRAP-seq and bulk RNA-seq from each tissue. $B$, Scatter plot of input and TRAP-seq shows high correlation between biological replicates for each approach. C, Empirical probability density function (PDF) of the TPM for all genes in analysis shows a similar distribution between replicates, which are each shown as a different color, for TRAP-seq and input. $\boldsymbol{D}$, Cumulative distribution of the fold change (FC) in input and TRAP-seq shows higher FCs in TRAP-seq samples. Kolgomorov-Smirnov test, ${ }^{* * *} p<0.001$.

the whole tissue transcriptional and $\mathrm{Nav}_{1.8}{ }^{+}$neuron translational landscapes between DRG and TG.

The first dimension of the clustering analysis identified clear differences between TG and DRG as well as distinctions within each subcluster comprised of the input (transcriptome) and TRAP (translatome) RNA sequencing (Fig. 2A). We observed strong correlation coefficients between biological replicates demonstrating low variability in the experimental protocol (Fig. 2B). Gene expression values (TPMs) were normalized to the 90th per- centile for each biological replicate, and the empirical probability density function of the normalized expression level (upper decile (ud)TPM) was plotted for the input and TRAP fractions (Fig. $2 C)$. The probability density function identified 2 peaks, and the inflection point was used to set the threshold expression values according to the sequencing depth (Fig. 2C). After further filtering, based on consistent expression among biological replicates, we included a total of 7358 genes in the final analysis to make comparisons between the DRG and TG transcriptomes and 
A

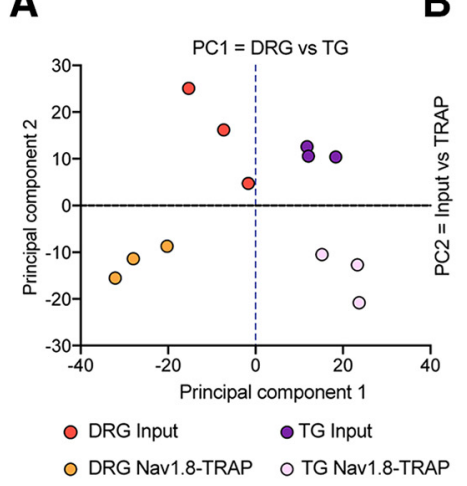

B

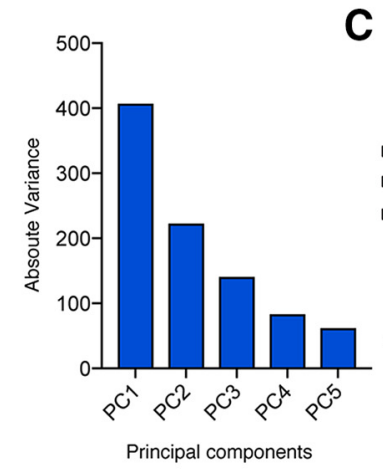

C

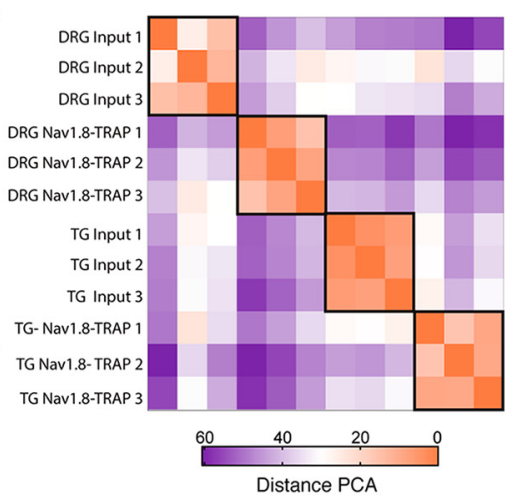

Figure 3. $P C$ analysis shows a clear difference between transcriptomes and translatomes in $T G$ and DRG. $A, P C$ analysis shows that differences between $T G$ and $D R G s$ whole tissue transcriptomes represent the first $P C$, whereas differences between transcriptome and translatome are the second $P C . B$, Absolute variances for each $P C$ show that $P C 1$ and $P C 2$ provide the majority of variation in the entire datasets. C, Heatmap of the absolute PC distances showing 4 distinct clusters, each of which is defined by whole transcriptome (input) versus TRAP-seq and the tissue.

A

Input

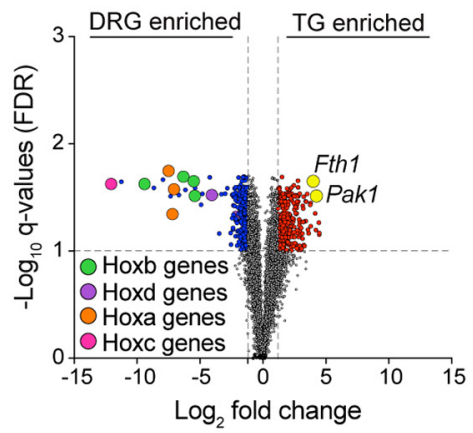

C

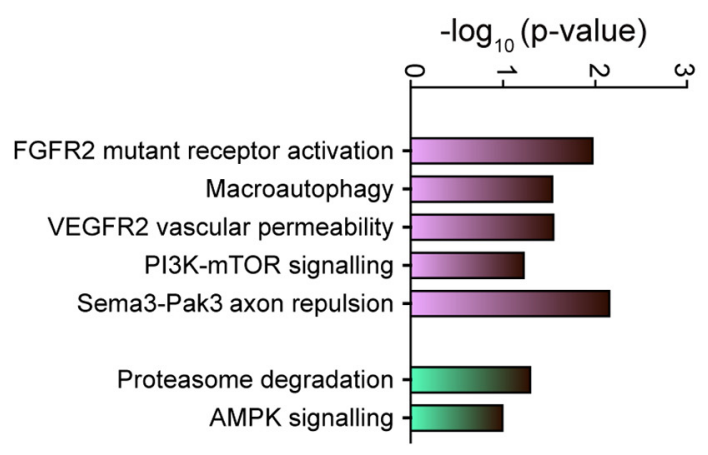

DRG enriched

TG enriched
Nav1.8-TRAP

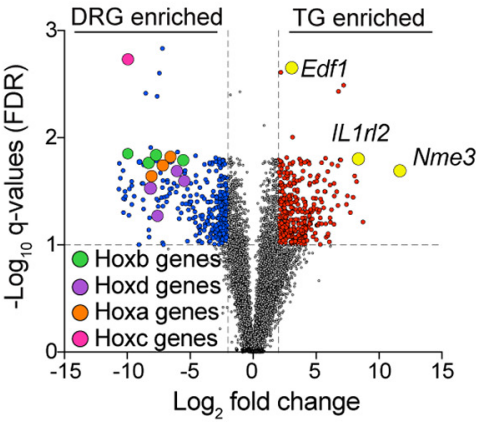

D

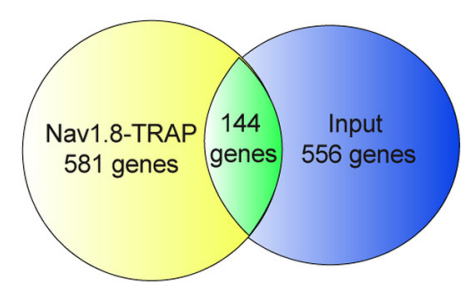

B

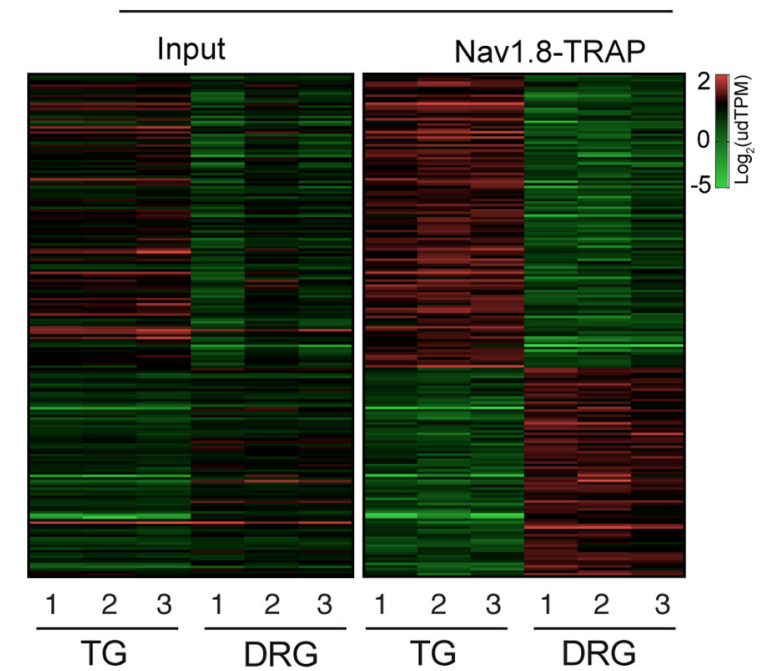

Figure 4. Transcriptomic and translatomic differences between the TG and DRG of mice. $\boldsymbol{A}, \boldsymbol{B}$, Volcano plots showing genes that are enriched in the DRG or TG in the whole tissue transcriptome (input) or in the TRAP-seq sample (Nav1.8-TRAP) with genes highlighted in the text labeled (yellow dots). C, G0 term analysis of the TRAP-seq-enriched mRNAs in DRG or TG using EnrichR (adjusted $p$ value $<0.05$ ) shows an enrichment in AMPK-related genes in the DRGs, whereas mTOR-related genes are highly translated in the TG. $D$, Heatmaps showing the expression level of enriched mRNAs (input) and enriched translated mRNAs (Nav1.8 TRAP) in both tissues showing discordance between the transcriptome and translatome mRNA levels.

Nav1.8-TRAP translatomes. Finally, we plotted the cumulative frequency distribution as a function of the log twofold change for each of these 7358 genes in TG and DRG biological replicates, and the 95th percentile was used to set the threshold fold change values for the input and TRAP fractions (Fig. 2D). Principal com- ponent (PC) analysis indicated that PC1 distinguished between TG and DRG, whereas PC2 detected a difference between input and Nav1.8-TRAP, suggesting a clear transcriptional and translational signature for both of these tissues (Fig. 3A). Detailed analysis of the variances for each PC clearly showed that the first 
Table 1. Genes upregulated in the TG input

\begin{tabular}{|c|c|c|c|c|c|}
\hline Genes & $\begin{array}{l}\text { Log2 fold } \\
\text { change }\end{array}$ & $p$ & $q$ & Genes & $\begin{array}{l}\text { Log2 fold } \\
\text { change }\end{array}$ \\
\hline 1700037C18Rik & 1.847 & 0.006 & 0.058 & Mrpl36 & 1.645 \\
\hline 6430548M08Rik & 1.249 & 0.000 & 0.026 & Mrpl44 & 1.331 \\
\hline 9130401M01Rik & 1.884 & 0.023 & 0.098 & Mrpl46 & 1.476 \\
\hline Aard & 2.059 & 0.001 & 0.030 & Mrps11 & 1.718 \\
\hline Abca2 & 1.917 & 0.000 & 0.026 & Mrps14 & 1.614 \\
\hline Abhd6 & 1.493 & 0.001 & 0.031 & Mrps23 & 1.332 \\
\hline Adarb1 & 1.426 & 0.011 & 0.072 & Mrps36 & 1.262 \\
\hline Adck2 & 1.969 & 0.004 & 0.049 & Mt2 & 3.017 \\
\hline$A k 5$ & 1.564 & 0.004 & 0.050 & $M t 3$ & 2.988 \\
\hline Alkbh3 & 2.585 & 0.002 & 0.039 & Mtap & 1.600 \\
\hline Amdhd2 & 1.234 & 0.001 & 0.030 & Mtfp1 & 1.528 \\
\hline Anapc13 & 1.952 & 0.003 & 0.046 & Mtmr4 & 1.402 \\
\hline Ap4s1 & 1.506 & 0.006 & 0.057 & $M x d 3$ & 1.297 \\
\hline$A p b b 1$ & 3.191 & 0.005 & 0.054 & Mxra8 & 2.100 \\
\hline Apip & 1.677 & 0.023 & 0.099 & Myl12a & 2.141 \\
\hline Apmap & 1.356 & 0.007 & 0.061 & Mylk & 1.285 \\
\hline Apod & 3.523 & 0.001 & 0.029 & Naa38 & 3.315 \\
\hline Apoe & 1.621 & 0.018 & 0.088 & Nap112 & 1.759 \\
\hline Arfip2 & 1.328 & 0.000 & 0.026 & $N d p$ & 1.418 \\
\hline Arhgef3 & 1.405 & 0.014 & 0.079 & Ndufa12 & 1.436 \\
\hline Arhgef4 & 1.255 & 0.005 & 0.055 & Ndufa13 & 1.921 \\
\hline Arih2 & 1.638 & 0.008 & 0.066 & Ndufb2 & 3.404 \\
\hline Armc5 & 1.203 & 0.020 & 0.093 & Necab3 & 1.663 \\
\hline $\operatorname{Arpc5I}$ & 1.248 & 0.021 & 0.095 & Nefh & 1.820 \\
\hline $\operatorname{Atp} 5 \mathrm{c} 1$ & 2.858 & 0.012 & 0.074 & Nif3/1 & 1.999 \\
\hline Atp5d & 2.923 & 0.004 & 0.052 & Nme3 & 3.589 \\
\hline Atp5j & 2.373 & 0.011 & 0.070 & Nnat & 1.368 \\
\hline Atp5sl & 1.335 & 0.005 & 0.053 & Nr2f6 & 1.334 \\
\hline Atxn7/3 & 1.365 & 0.015 & 0.080 & Nsg1 & 2.505 \\
\hline Avpi1 & 1.810 & 0.015 & 0.082 & Nsmaf & 1.511 \\
\hline B930041F14Rik & 2.887 & 0.004 & 0.051 & Nubp2 & 2.798 \\
\hline Bad & 1.797 & 0.005 & 0.052 & Nudt1 & 1.395 \\
\hline Bet1I & 1.486 & 0.014 & 0.079 & Nudt13 & 1.358 \\
\hline Bod1 & 1.529 & 0.013 & 0.077 & Odc1 & 2.129 \\
\hline Cacng5 & 2.058 & 0.008 & 0.063 & Otud3 & 2.021 \\
\hline Calb2 & 3.253 & 0.005 & 0.053 & P2rx6 & 1.581 \\
\hline Calu & 1.213 & 0.001 & 0.031 & Pacs2 & 1.388 \\
\hline Camkk1 & 1.514 & 0.013 & 0.076 & Pak1 & 3.982 \\
\hline Casp3 & 1.506 & 0.002 & 0.035 & Pard6a & 1.806 \\
\hline$C b \times 7$ & 1.324 & 0.015 & 0.081 & Pced1a & 1.460 \\
\hline Ccdc12 & 1.344 & 0.018 & 0.087 & Pcp4l1 & 1.512 \\
\hline Ccdc124 & 1.505 & 0.015 & 0.082 & Pdia4 & 1.410 \\
\hline Ccdc63 & 3.251 & 0.018 & 0.088 & Pdlim2 & 2.857 \\
\hline$C d 81$ & 1.413 & 0.005 & 0.055 & Pex11b & 2.281 \\
\hline$C d a$ & 1.665 & 0.003 & 0.044 & Pgbd5 & 1.688 \\
\hline Cdc37 & 1.442 & 0.014 & 0.079 & Pin1 & 1.948 \\
\hline$C d k 5 r 1$ & 1.482 & 0.005 & 0.055 & Pkdcc & 1.610 \\
\hline Cdpf1 & 1.216 & 0.014 & 0.079 & Pkm & 1.408 \\
\hline Cdr2l & 1.629 & 0.015 & 0.082 & Pla2g16 & 2.793 \\
\hline Cela1 & 1.982 & 0.014 & 0.080 & Pled4 & 1.204 \\
\hline Cenpf & 3.061 & 0.020 & 0.091 & Plekha4 & 1.487 \\
\hline Cep19 & 1.321 & 0.005 & 0.053 & Plk5 & 2.167 \\
\hline Cgrrf1 & 2.813 & 0.002 & 0.035 & Pllp & 1.905 \\
\hline Chchd1 & 2.920 & 0.001 & 0.029 & Plpp1 & 2.148 \\
\hline Chchd3 & 1.514 & 0.018 & 0.088 & Plxdc1 & 1.659 \\
\hline Chga & 1.707 & 0.007 & 0.063 & Pnpla2 & 1.296 \\
\hline Chgb & 1.656 & 0.002 & 0.039 & Polr $2 b$ & 1.215 \\
\hline Chmp6 & 2.654 & 0.003 & 0.045 & Polr2l & 4.483 \\
\hline Chpf2 & 1.312 & 0.015 & 0.082 & Pon2 & 1.207 \\
\hline Chrac1 & 1.481 & 0.002 & 0.035 & Ppa2 & 1.552 \\
\hline Ckmt1 & 1.257 & 0.020 & 0.093 & Ppdpf & 1.387 \\
\hline Clcn7 & 1.490 & 0.005 & 0.053 & Ppfia4 & 2.311 \\
\hline Clec2l & 2.448 & 0.002 & 0.036 & Ppm1f & 1.386 \\
\hline $\mathrm{Clu}$ & 1.283 & 0.000 & 0.029 & Ppp1r16b & 1.798 \\
\hline
\end{tabular}

Table 1. Continued

\begin{tabular}{|c|c|c|c|c|c|c|c|}
\hline Genes & $\begin{array}{l}\text { Log2 fold } \\
\text { change }\end{array}$ & $p$ & $q$ & Genes & $\begin{array}{l}\text { Log2 fold } \\
\text { change }\end{array}$ & $p$ & $q$ \\
\hline Clybl & 1.665 & 0.015 & 0.081 & Ppp2r4 & 1.920 & 0.003 & 0.044 \\
\hline Cnnm4 & 1.420 & 0.022 & 0.097 & Prorsd1 & 1.630 & 0.018 & 0.088 \\
\hline Cnp & 2.467 & 0.000 & 0.024 & Prpsap1 & 1.397 & 0.001 & 0.031 \\
\hline Cnpy3 & 1.844 & 0.008 & 0.063 & Prss 12 & 2.286 & 0.000 & 0.029 \\
\hline Cnst & 1.991 & 0.017 & 0.085 & Prx & 1.489 & 0.001 & 0.033 \\
\hline Cops3 & 1.363 & 0.008 & 0.064 & Psmb11 & 2.692 & 0.021 & 0.094 \\
\hline Coq10a & 2.084 & 0.010 & 0.068 & Psmb7 & 2.699 & 0.003 & 0.045 \\
\hline Cot/1 & 1.523 & 0.002 & 0.035 & Ptcd2 & 1.699 & 0.016 & 0.085 \\
\hline Cox6b1 & 1.564 & 0.018 & 0.088 & Ptgds & 1.888 & 0.008 & 0.063 \\
\hline Cox7a2l & 1.430 & 0.005 & 0.053 & Rab11fip5 & 1.791 & 0.008 & 0.063 \\
\hline Cplx1 & 1.869 & 0.008 & 0.065 & Rab35 & 1.527 & 0.008 & 0.063 \\
\hline Crip1 & 1.529 & 0.014 & 0.079 & Rab3ip & 1.739 & 0.005 & 0.053 \\
\hline Crispld2 & 1.532 & 0.006 & 0.056 & Rad54l & 1.412 & 0.018 & 0.088 \\
\hline Cspg5 & 1.427 & 0.010 & 0.069 & Rarres1 & 1.432 & 0.001 & 0.031 \\
\hline Csrp2 & 2.150 & 0.001 & 0.030 & Rcor2 & 1.946 & 0.006 & 0.056 \\
\hline Cst3 & 1.798 & 0.004 & 0.048 & Rep15 & 3.803 & 0.006 & 0.059 \\
\hline Ctif & 1.444 & 0.023 & 0.099 & Rhbdd2 & 1.301 & 0.002 & 0.035 \\
\hline Ctnnbl1 & 1.636 & 0.019 & 0.089 & Rhot2 & 1.367 & 0.013 & 0.076 \\
\hline Ctsf & 1.591 & 0.000 & 0.029 & Rimklb & 1.410 & 0.021 & 0.094 \\
\hline Cyb5a & 1.289 & 0.009 & 0.068 & Rnaseh2c & 2.276 & 0.010 & 0.069 \\
\hline Cyc1 & 3.399 & 0.002 & 0.034 & Rnf114 & 1.743 & 0.001 & 0.029 \\
\hline$D b i$ & 1.474 & 0.015 & 0.081 & Rnf121 & 1.390 & 0.005 & 0.053 \\
\hline Dexi & 2.255 & 0.000 & 0.026 & Rnf157 & 1.611 & 0.005 & 0.055 \\
\hline Dffa & 1.337 & 0.011 & 0.072 & Rom1 & 2.221 & 0.000 & 0.032 \\
\hline Dhdh & 4.211 & 0.008 & 0.064 & Rpl10a & 2.066 & 0.008 & 0.063 \\
\hline$D \lg 2$ & 1.409 & 0.004 & 0.048 & Rprm & 1.661 & 0.004 & 0.048 \\
\hline Dnajb9 & 1.633 & 0.001 & 0.030 & Rps27 & 2.271 & 0.023 & 0.099 \\
\hline Dnajc11 & 1.379 & 0.004 & 0.050 & S100a4 & 1.233 & 0.003 & 0.045 \\
\hline Dnal4 & 1.470 & 0.019 & 0.090 & $S a c 3 d 1$ & 3.758 & 0.002 & 0.037 \\
\hline Dpm3 & 2.497 & 0.012 & 0.074 & Sap18 & 1.448 & 0.024 & 0.100 \\
\hline Dpp9 & 1.459 & 0.007 & 0.061 & Sat1 & 1.390 & 0.005 & 0.055 \\
\hline Eaf1 & 1.246 & 0.002 & 0.035 & $\operatorname{Scg} 5$ & 1.738 & 0.011 & 0.072 \\
\hline Edf1 & 2.374 & 0.017 & 0.086 & $\operatorname{Scn} 4 b$ & 1.595 & 0.005 & 0.052 \\
\hline Efcc1 & 4.477 & 0.009 & 0.068 & Scrn1 & 1.936 & 0.001 & 0.030 \\
\hline Egln2 & 1.685 & 0.001 & 0.031 & $S C x$ & 2.374 & 0.004 & 0.049 \\
\hline Eif2b2 & 2.535 & 0.001 & 0.031 & Scyl3 & 3.634 & 0.010 & 0.068 \\
\hline Eif3I & 2.072 & 0.012 & 0.075 & $\operatorname{Sec} 13$ & 2.615 & 0.017 & 0.086 \\
\hline Elp3 & 1.598 & 0.001 & 0.031 & Selm & 2.482 & 0.009 & 0.067 \\
\hline Eme1 & 1.587 & 0.009 & 0.068 & Sepp1 & 2.699 & 0.002 & 0.035 \\
\hline Eme2 & 1.501 & 0.006 & 0.056 & Sfxn5 & 1.714 & 0.003 & 0.046 \\
\hline Endod1 & 1.402 & 0.002 & 0.034 & Sh3bgr & 1.391 & 0.024 & 0.100 \\
\hline Enho & 1.447 & 0.001 & 0.031 & $S h 3 g / 2$ & 1.278 & 0.010 & 0.069 \\
\hline Eno2 & 1.548 & 0.020 & 0.092 & Sh3rf1 & 1.571 & 0.002 & 0.038 \\
\hline Eny2 & 1.328 & 0.009 & 0.067 & Shd & 1.737 & 0.002 & 0.038 \\
\hline Epn3 & 1.200 & 0.014 & 0.079 & Sirt2 & 1.287 & 0.004 & 0.050 \\
\hline Esrrg & 1.705 & 0.007 & 0.063 & SIc22a17 & 4.451 & 0.005 & 0.054 \\
\hline$E t / 4$ & 1.537 & 0.005 & 0.052 & SIC25a25 & 1.508 & 0.015 & 0.082 \\
\hline Fabp3 & 1.275 & 0.022 & 0.096 & SIC25a43 & 3.074 & 0.021 & 0.095 \\
\hline Fabp7 & 3.165 & 0.002 & 0.038 & SIC $25 a 5$ & 3.786 & 0.002 & 0.036 \\
\hline Faim2 & 1.328 & 0.002 & 0.034 & Slc38a10 & 1.399 & 0.017 & 0.087 \\
\hline Fam $160 b 2$ & 1.237 & 0.015 & 0.083 & SIc4a2 & 1.322 & 0.014 & 0.079 \\
\hline Fam162a & 2.032 & 0.005 & 0.054 & Slc6a8 & 1.844 & 0.008 & 0.063 \\
\hline Fam19a5 & 2.083 & 0.016 & 0.084 & Slc9a3r1 & 2.002 & 0.004 & 0.051 \\
\hline Fam57b & 2.035 & 0.005 & 0.054 & Slco2b1 & 2.443 & 0.004 & 0.051 \\
\hline Fars2 & 1.969 & 0.002 & 0.038 & Smim1 & 1.230 & 0.009 & 0.066 \\
\hline Fbx/12 & 1.201 & 0.006 & 0.057 & Smim4 & 1.838 & 0.002 & 0.035 \\
\hline$F b \times 027$ & 2.483 & 0.001 & 0.031 & Smoc2 & 1.302 & 0.004 & 0.049 \\
\hline Fbxo44 & 1.807 & 0.004 & 0.048 & Smox & 2.826 & 0.001 & 0.030 \\
\hline Fchsd1 & 1.429 & 0.005 & 0.053 & Smpx & 1.427 & 0.001 & 0.034 \\
\hline Fdx1l & 1.385 & 0.018 & 0.088 & Sncb & 1.711 & 0.007 & 0.060 \\
\hline Fhdc1 & 1.306 & 0.000 & 0.026 & Snn & 2.308 & 0.012 & 0.074 \\
\hline Fkbp2 & 1.980 & 0.015 & 0.083 & Snx 22 & 2.722 & 0.009 & 0.068 \\
\hline Fkbp4 & 1.407 & 0.005 & 0.055 & Sphkap & 2.242 & 0.001 & 0.030 \\
\hline \multirow[t]{2}{*}{ Fth1 } & 4.168 & 0.000 & 0.022 & Sptb & 1.567 & 0.020 & 0.093 \\
\hline & & & & & & \multicolumn{2}{|c|}{ (Table continues) } \\
\hline
\end{tabular}


Table 1. Continued

\begin{tabular}{|c|c|c|c|c|c|c|c|}
\hline Genes & $\begin{array}{l}\text { Log2 fold } \\
\text { change }\end{array}$ & $p$ & $q$ & Genes & $\begin{array}{l}\text { Log2 fold } \\
\text { change }\end{array}$ & $p$ & $q$ \\
\hline Fuca1 & 1.308 & 0.013 & 0.076 & Srm & 1.623 & 0.012 & 0.075 \\
\hline Gatb & 1.370 & 0.008 & 0.064 & Stard3 & 1.798 & 0.002 & 0.039 \\
\hline Glb112 & 2.686 & 0.001 & 0.031 & Stk32c & 1.467 & 0.022 & 0.096 \\
\hline Gle1 & 1.391 & 0.020 & 0.091 & Stmn4 & 2.074 & 0.000 & 0.028 \\
\hline Glyr1 & 1.263 & 0.014 & 0.079 & Stxbp6 & 1.486 & 0.024 & 0.100 \\
\hline Gps1 & 2.939 & 0.008 & 0.065 & Suclg1 & 2.146 & 0.008 & 0.064 \\
\hline Gpx1 & 1.719 & 0.023 & 0.097 & Supt4a & 3.247 & 0.001 & 0.031 \\
\hline Grk6 & 1.513 & 0.007 & 0.061 & Suv420h1 & 2.426 & 0.019 & 0.090 \\
\hline Gtf2h4 & 4.152 & 0.003 & 0.045 & Syn2 & 1.312 & 0.001 & 0.030 \\
\hline Gtf2i & 1.710 & 0.003 & 0.045 & Syne4 & 2.723 & 0.002 & 0.037 \\
\hline Gtf2ird1 & 2.143 & 0.010 & 0.069 & Sys1 & 1.933 & 0.003 & 0.046 \\
\hline Haghl & 1.514 & 0.008 & 0.063 & Taf6l & 1.539 & 0.006 & 0.056 \\
\hline Hapln4 & 1.571 & 0.012 & 0.075 & Tango2 & 1.243 & 0.008 & 0.065 \\
\hline Harbi1 & 1.772 & 0.011 & 0.070 & Tecr & 1.980 & 0.024 & 0.100 \\
\hline Haus8 & 3.145 & 0.010 & 0.069 & $T f b 1 m$ & 1.304 & 0.012 & 0.073 \\
\hline Hax1 & 1.463 & 0.007 & 0.063 & Thap11 & 1.324 & 0.004 & 0.049 \\
\hline Hebp2 & 1.567 & 0.001 & 0.033 & Tifab & 1.602 & 0.018 & 0.088 \\
\hline Hhatl & 2.106 & 0.003 & 0.045 & Timm 9 & 1.715 & 0.009 & 0.066 \\
\hline Hid1 & 1.773 & 0.013 & 0.077 & Tmcol & 1.240 & 0.014 & 0.079 \\
\hline Hist3h2ba & 1.307 & 0.005 & 0.052 & Tmem101 & 1.649 & 0.020 & 0.092 \\
\hline Hlcs & 1.772 & 0.012 & 0.075 & Tmem126a & 1.893 & 0.001 & 0.030 \\
\hline Homer3 & 2.804 & 0.001 & 0.031 & Tmem132c & 2.205 & 0.007 & 0.059 \\
\hline Hрса & 1.469 & 0.003 & 0.046 & Tmem14c & 1.461 & 0.022 & 0.096 \\
\hline Hs 3st1 & 1.520 & 0.012 & 0.075 & Tmem18 & 1.588 & 0.002 & 0.040 \\
\hline$H s d l 2$ & 1.375 & 0.005 & 0.053 & Tmem201 & 1.336 & 0.001 & 0.030 \\
\hline Htra1 & 2.177 & 0.007 & 0.063 & Tmem203 & 1.637 & 0.018 & 0.087 \\
\hline Hunk & 3.047 & 0.007 & 0.061 & Tmem229b & 1.572 & 0.002 & 0.038 \\
\hline Iba57 & 1.357 & 0.014 & 0.080 & Tmem242 & 1.386 & 0.018 & 0.088 \\
\hline Id 3 & 2.405 & 0.005 & 0.053 & $\operatorname{Tmem} 25$ & 2.039 & 0.001 & 0.030 \\
\hline Idh2 & 1.732 & 0.002 & 0.036 & Tmem258 & 3.156 & 0.013 & 0.076 \\
\hline$I d h 3 b$ & 1.848 & 0.007 & 0.062 & Tmem60 & 1.268 & 0.001 & 0.031 \\
\hline Imp3 & 2.481 & 0.007 & 0.060 & Tnfrsf1a & 1.468 & 0.005 & 0.055 \\
\hline Impdh2 & 1.694 & 0.004 & 0.051 & Tpbgl & 2.734 & 0.008 & 0.064 \\
\hline Inpp5j & 2.890 & 0.006 & 0.058 & Trak1 & 1.435 & 0.005 & 0.053 \\
\hline Itih5 & 1.235 & 0.006 & 0.058 & Trappc3 & 2.247 & 0.020 & 0.093 \\
\hline $\operatorname{Itm} 2 \mathrm{C}$ & 1.551 & 0.002 & 0.035 & Trf & 2.565 & 0.002 & 0.037 \\
\hline Jam3 & 2.138 & 0.000 & 0.030 & Trp53rka & 1.316 & 0.001 & 0.030 \\
\hline Kat2a & 1.756 & 0.009 & 0.067 & Tspan3 & 1.262 & 0.012 & 0.074 \\
\hline Kenq4 & 2.581 & 0.001 & 0.030 & Ttc9b & 1.870 & 0.011 & 0.071 \\
\hline Kctd15 & 2.542 & 0.016 & 0.084 & $T x n 14 b$ & 1.574 & 0.003 & 0.044 \\
\hline Krt10 & 1.758 & 0.008 & 0.064 & Tyr & 2.829 & 0.023 & 0.097 \\
\hline Land1 & 1.907 & 0.000 & 0.029 & Tyro3 & 2.928 & 0.019 & 0.089 \\
\hline Laptm4b & 1.441 & 0.001 & 0.031 & U2af114 & 1.981 & 0.002 & 0.035 \\
\hline$L d h b$ & 1.497 & 0.014 & 0.080 & Ube2v1 & 1.615 & 0.006 & 0.058 \\
\hline Letm1 & 1.305 & 0.015 & 0.081 & Ubl5 & 1.577 & 0.021 & 0.093 \\
\hline Lgi3 & 1.359 & 0.006 & 0.055 & Ufsp1 & 2.295 & 0.001 & 0.034 \\
\hline Limd1 & 1.389 & 0.000 & 0.028 & Ulk1 & 1.439 & 0.008 & 0.064 \\
\hline Lrp1 & 1.242 & 0.001 & 0.033 & Uqce2 & 2.331 & 0.006 & 0.058 \\
\hline Lyz2 & 3.211 & 0.002 & 0.040 & Uqce3 & 1.376 & 0.005 & 0.054 \\
\hline Lztr1 & 1.525 & 0.018 & 0.088 & Uqcrh & 1.253 & 0.016 & 0.084 \\
\hline Maged2 & 1.849 & 0.012 & 0.074 & Vasp & 1.204 & 0.024 & 0.100 \\
\hline Map1/c3b & 1.800 & 0.001 & 0.030 & Vim & 1.384 & 0.007 & 0.060 \\
\hline Mark4 & 2.680 & 0.002 & 0.040 & Vwa7 & 1.728 & 0.001 & 0.033 \\
\hline Mars & 1.517 & 0.003 & 0.046 & Wbp1 & 1.987 & 0.009 & 0.068 \\
\hline Mat2a & 1.570 & 0.001 & 0.030 & Wfs1 & 2.149 & 0.001 & 0.031 \\
\hline Meis2 & 1.776 & 0.004 & 0.051 & Wwox & 1.523 & 0.023 & 0.098 \\
\hline Mgat5 & 1.417 & 0.001 & 0.032 & Yif1a & 1.236 & 0.006 & 0.059 \\
\hline Mgst3 & 3.432 & 0.001 & 0.035 & Zfand $2 b$ & 2.929 & 0.005 & 0.054 \\
\hline Mief1 & 1.479 & 0.013 & 0.078 & Zfp180 & 1.595 & 0.014 & 0.080 \\
\hline Mmd2 & 2.595 & 0.003 & 0.044 & Zfp335 & 1.645 & 0.017 & 0.085 \\
\hline Mobp & 1.996 & 0.015 & 0.082 & Zfp771 & 1.604 & 0.023 & 0.097 \\
\hline Mpc2 & 1.877 & 0.004 & 0.052 & & & & \\
\hline
\end{tabular}

Table 2. Genes upregulated in the DRG input

\begin{tabular}{|c|c|c|c|c|c|c|c|}
\hline Genes & $\begin{array}{l}\text { Log2 fold } \\
\text { change }\end{array}$ & $p$ & $q$ & Genes & $\begin{array}{l}\text { Log2 fold } \\
\text { change }\end{array}$ & $p$ & $q$ \\
\hline 1700019D03Rik & -1.367 & 0.001 & 0.030 & Mpped2 & -2.004 & 0.000 & 0.04 \\
\hline 9330159F19Rik & -1.337 & 0.001 & 0.031 & Mpv1712 & -1.509 & 0.006 & 0.055 \\
\hline 9330182L06Rik & -1.668 & 0.006 & 0.056 & Mt-C03 & -1.553 & 0.007 & 0.063 \\
\hline$A b c a 5$ & -1.456 & 0.008 & 0.063 & Myc & -1.925 & 0.001 & 0.030 \\
\hline Acacb & -1.267 & 0.010 & 0.070 & Myh1 & -4.915 & 0.002 & 0.037 \\
\hline Acbd5 & -1.220 & 0.018 & 0.088 & Myl1 & -7.949 & 0.000 & 0.022 \\
\hline Acpp & -1.662 & 0.000 & 0.022 & Myo1b & -1.242 & 0.004 & 0.049 \\
\hline Acs/4 & -1.505 & 0.000 & 0.025 & Myom1 & -2.336 & 0.002 & 0.038 \\
\hline Acta1 & -6.161 & 0.000 & 0.027 & Myt11 & -1.439 & 0.005 & 0.055 \\
\hline Actn1 & -1.467 & 0.002 & 0.036 & Nectin1 & -1.609 & 0.010 & 0.069 \\
\hline Adcyap1 & -1.814 & 0.003 & 0.045 & Nedd4I & -1.349 & 0.001 & 0.029 \\
\hline Adgrd1 & -1.531 & 0.006 & 0.057 & Nek1 & -1.475 & 0.017 & 0.086 \\
\hline Adgrf5 & -1.678 & 0.004 & 0.049 & Nfya & -1.752 & 0.018 & 0.087 \\
\hline Adk & -2.250 & 0.000 & 0.026 & Nhs & -1.233 & 0.001 & 0.03 \\
\hline Agtr1a & -2.244 & 0.002 & 0.038 & Nktr & -1.403 & 0.008 & 0.063 \\
\hline Ammecr1 & -1.266 & 0.012 & 0.074 & Noct & -1.573 & 0.015 & 0.082 \\
\hline Ank3 & -1.585 & 0.000 & 0.029 & Nptx2 & -1.297 & 0.002 & 0.040 \\
\hline Ankrd6 & -1.453 & 0.003 & 0.043 & Nptxr & -1.481 & 0.001 & 0.031 \\
\hline Ano3 & -1.205 & 0.003 & 0.044 & Npy2r & -2.372 & 0.007 & 0.061 \\
\hline Arfgef2 & -1.305 & 0.000 & 0.026 & Nras & -1.633 & 0.012 & 0.075 \\
\hline Arhgap23 & -1.415 & 0.012 & 0.074 & Nrip1 & -1.304 & 0.000 & 0.027 \\
\hline Arhgap26 & -2.827 & 0.000 & 0.026 & Nrxn3 & -1.701 & 0.008 & 0.064 \\
\hline Arrdc3 & -1.213 & 0.023 & 0.099 & Nt5e & -2.145 & 0.001 & 0.034 \\
\hline Ass1 & -1.757 & 0.002 & 0.040 & Nup88 & -1.244 & 0.023 & 0.098 \\
\hline Astn1 & -1.500 & 0.007 & 0.061 & $\mathrm{OCrl}$ & -1.219 & 0.004 & 0.049 \\
\hline Atp2b4 & -2.126 & 0.001 & 0.030 & Ormdl1 & -1.558 & 0.004 & 0.048 \\
\hline Auts2 & -1.836 & 0.000 & 0.027 & Osbpl3 & -1.696 & 0.000 & 0.026 \\
\hline B630005N14Rik & -1.380 & 0.017 & 0.086 & Pabpc1 & -1.654 & 0.020 & 0.093 \\
\hline Bdnf & -1.450 & 0.003 & 0.042 & Pabpn1 & -1.450 & 0.009 & 0.067 \\
\hline Bnc2 & -1.401 & 0.004 & 0.050 & Palm2 & -2.056 & 0.005 & 0.055 \\
\hline Brms11 & -1.357 & & 0.073 & Palmd & -1.212 & 0 & 0.026 \\
\hline Cacna2d1 & -1.978 & 0.000 & 0.039 & Pam & -1.491 & 06 & $05 /$ \\
\hline Camk2a & -1.912 & 0.001 & 0.031 & Panx1 & -1.364 & 0.002 & 0.040 \\
\hline Camk2d & -1.608 & 0.011 & 0.071 & Paqr3 & -1.230 & 0.006 & 0.058 \\
\hline Camta1 & -1.640 & 0.013 & 0.077 & Pde10a & -1.289 & 0.001 & 0.030 \\
\hline Capn1 & -1.429 & 0.003 & 0.042 & Pde11a & -1.938 & 0.000 & 0.02 \\
\hline Car8 & -1.627 & 0.011 & 0.072 & Pdlim1 & -1.524 & 0.005 & 0.05 \\
\hline Casz1 & -1 & 0.004 & 0.048 & Pfkp & 96 & 4 & 05 \\
\hline Cedc141 & -1.908 & 0.002 & 0.035 & Pfn1 & -2.156 & 0.001 & 0.03 \\
\hline Cct8 & -1.364 & 0.009 & 0.068 & Pgm2/1 & -1.494 & 0.000 & 0.02 \\
\hline$C d 274$ & -1.797 & 0.005 & 0.052 & Phip & -1.266 & 0.004 & 0.05 \\
\hline Cd2ap & -1.457 & 0.012 & 0.074 & Pitpnc1 & -1.209 & 0.000 & 0.02 \\
\hline$C d 44$ & -1.559 & 0.001 & 0.030 & Pitpnm2 & -1.297 & 0.015 & 0.08 \\
\hline$C d 47$ & -1.588 & 0.000 & 0.020 & Pkia & -1.817 & 0.003 & 0.04 \\
\hline$C d 55$ & -2.309 & 0.001 & 0.031 & Plcb3 & -1.560 & 0.000 & 0.02 \\
\hline$C d c 14 b$ & -1.372 & 0.000 & 0.025 & Plekha6 & -1.243 & 0.008 & 0.06 \\
\hline Celf4 & -1.240 & 0.005 & 0.052 & Plvap & -1.298 & 0.018 & 0.087 \\
\hline Celf6 & -1.775 & 0.013 & 0.077 & Plxnc1 & -1.740 & 0.005 & 0.05 \\
\hline Cep170 & -1.468 & 0.006 & 0.058 & Plxnd1 & -1.345 & 0.002 & 0.03 \\
\hline Cfap157 & -1.378 & 0.002 & 0.040 & Polrza & -1 & 1 & 0.03 \\
\hline $\mathrm{Chml}$ & -1.782 & 0.012 & 0.074 & Pou1f1 & -2.038 & 0.019 & 0.08 \\
\hline Chpt1 & -1.408 & 0.010 & 0.068 & Ppef1 & -1.257 & 0.004 & 0.04 \\
\hline Ciapin1 & -1.263 & 0.020 & 0.093 & Ppp1r12a & -1.564 & 0.001 & 0.030 \\
\hline $\mathrm{Ckm}$ & -7.336 & 0.001 & 0.031 & Рррзса & -1.498 & 0.000 & 0.02 \\
\hline Clgn & -1.231 & 0.009 & 0.067 & Ppp6c & -1.218 & 0.010 & 0.06 \\
\hline Clip2 & -2.335 & 0.003 & 0.046 & Prdm8 & -2.355 & 0.001 & 0.03 \\
\hline Cmip & -1.580 & 0.002 & 0.037 & $\operatorname{Prg} 2$ & -3.717 & 0.001 & 0.03 \\
\hline Cnot1 & -1.720 & 0.000 & 0.025 & Prkag2 & -1.331 & 0.022 & 0.09 \\
\hline Cnot4 & -1.280 & 0.004 & 0.048 & Prkar2b & -1.651 & 0.000 & 0.02 \\
\hline Cntrl & -1.361 & 0.004 & 0.050 & Prkca & -2.159 & 0.000 & 0.02 \\
\hline Cpeb1 & -1.200 & 0.005 & 0.053 & Ptgdr & -1.577 & 0.002 & 0.03 \\
\hline Cpne2 & -1.790 & 0.018 & 0.087 & Ptger1 & -1.387 & 0.008 & 0 \\
\hline Cpsf7 & -1.260 & 0.010 & 0.069 & Ptms & -1.523 & 0.000 & 0.02 \\
\hline Csrnp3 & -1.215 & 0.000 & 0.025 & Ptprt & -2.355 & 0.001 & 0.029 \\
\hline
\end{tabular}


Table 2. Continued

\begin{tabular}{|c|c|c|c|c|c|c|c|}
\hline Genes & $\begin{array}{l}\text { Log2 fold } \\
\text { change }\end{array}$ & $p$ & $q$ & Genes & $\begin{array}{l}\text { Log2 fold } \\
\text { change }\end{array}$ & $p$ & $q$ \\
\hline Ctsl & 1.450 & 0.002 & 0.034 & Ptrf & -1.688 & 0.002 & 0.040 \\
\hline$D d x 3 x$ & 1.330 & 0.001 & 0.030 & Pum1 & -1.405 & 0.008 & 0.065 \\
\hline Deptor & -1.652 & 0.001 & 0.033 & Pura & -1.301 & 0.006 & 0.056 \\
\hline Dgkh & -1.341 & 0.005 & 0.055 & Purb & -1.209 & 0.021 & 0.095 \\
\hline Dgkz & -1.775 & 0.000 & 0.024 & Pygl & -1.338 & 0.001 & 0.031 \\
\hline Disp2 & -1.423 & 0.010 & 0.069 & Rab27b & -1.526 & 0.016 & 0.083 \\
\hline Dpp10 & -1.486 & 0.000 & 0.021 & Rab39b & -1.515 & 0.003 & 0.045 \\
\hline Dpp6 & -1.293 & 0.002 & 0.039 & Rab3c & -1.439 & 0.013 & 0.075 \\
\hline Ebf3 & -1.919 & 0.002 & 0.035 & Rabgap1l & -1.317 & 0.003 & 0.044 \\
\hline Eif4e3 & -1.421 & 0.001 & 0.032 & Raph1 & -1.327 & 0.007 & 0.060 \\
\hline Etnk1 & -1.896 & 0.001 & 0.030 & Rasgrp1 & -1.547 & 0.001 & 0.031 \\
\hline$F 2 r 12$ & -1.621 & 0.012 & 0.075 & Rbms1 & -1 & 0.002 & 0.034 \\
\hline Fabp4 & -5.383 & 0.000 & 0.026 & Reps2 & -1 & 0.003 & 0.041 \\
\hline Fam102b & -1.588 & 0.000 & 0.025 & Rgmb & -1 & 0.015 & 0.082 \\
\hline Fam122b & -2.137 & 0.004 & 0.050 & Rgs 17 & -2 & 0.000 & 0.026 \\
\hline Fam179b & -1.540 & 0.003 & 0.041 & Rnf144a & -1 & 0.000 & 0.029 \\
\hline Fam214b & -1.405 & 001 & 0.031 & Robo2 & -1 & 0.001 & 0.030 \\
\hline Fam222b & -1.668 & 007 & 0.062 & Rps11 & -1 & 0.005 & 0.055 \\
\hline Filip1 & -1.223 & 0.001 & 0.030 & Rspo2 & -1 & 0.000 & 0.026 \\
\hline Fsd2 & -2.160 & 012 & 0.073 & Runx1 & -1 & 0.006 & 0.055 \\
\hline Gal & -2.045 & 002 & 0.035 & Ryr1 & , & 0.010 & 0.069 \\
\hline Ghr & -2.439 & .004 & 0.049 & S100a11 & -2 & 0.007 & 0.063 \\
\hline Gm17305 & -1.767 & 0.003 & 0.046 & $5100 a 8$ & -3 & 0.001 & 0.029 \\
\hline Gm42417 & -4.801 & 000 & 0.029 & 510 & -4 & 0.000 & 0.021 \\
\hline Gmfb & -1.259 & & & Safb & -1 & 0.011 & 0.070 \\
\hline Gna14 & -1.468 & & & Samsr & -2 & 0.001 & 0.030 \\
\hline Gnai3 & -1.748 & 1 & 0.072 & Scd1 & -1 & 0.001 & 0.034 \\
\hline Gnao1 & -1.462 & 001 & 0.032 & $\operatorname{Scg} 2$ & -1 & 0.009 & 0.066 \\
\hline Gnaq & -1.531 & 002 & 0.034 & $\operatorname{Scg} 3$ & -1 & 0.002 & 0.038 \\
\hline Gnb4 & -1.236 & 008 & 0.064 & $\sin 9 a$ & -1 & 0.000 & 0.027 \\
\hline Gp1bb & -1.271 & & & Scyl2 & & & 0.031 \\
\hline Grip1 & -1.423 & & 0.076 & $S d c b p$ & -1 & 1 & 0.030 \\
\hline Grm7 & -1.784 & 01 & 0.033 & Sema $4 b$ & -1 & 0.002 & 0.039 \\
\hline$H 2-K 1$ & -1.611 & 019 & 0.091 & Sepw1 & -1 & 0.013 & 0.077 \\
\hline Hace1 & -1.481 & 008 & 0.065 & Slc16a3 & -2 & 0.001 & 0.029 \\
\hline $\mathrm{Hba}-\mathrm{a} 2$ & -1.762 & .004 & 0.052 & SIC27a3 & -1 & 0.008 & 0.065 \\
\hline$H b b-b t$ & -1.668 & 0.004 & 0.052 & $S / c 35 a 5$ & -1 & 0.000 & 0.038 \\
\hline Hen3 & -1.929 & & & SIC37a1 & & & 0.085 \\
\hline Hgf & -1.762 & & & $S / c 3$ & & 0.003 & 0.041 \\
\hline Hmbox1 & -1.450 & 0.011 & 0.072 & SIC51a & -1 & 0.002 & 0.035 \\
\hline $\mathrm{Hmgd}$ & -1.314 & 013 & 0.077 & Slc5a3 & -1 & 0.014 & 0.080 \\
\hline Hoxa10 & -7.016 & 000 & 0.020 & Slc9a6 & -1 & 0.002 & 0.039 \\
\hline Hoxa7 & -7.044 & 000 & 0.045 & Smim1011 & -1 & 0.002 & 0.036 \\
\hline Hoxa9 & -6.931 & & 0.027 & Smim5 & -1 & 0. & 0.080 \\
\hline Hoxb2 & -5.272 & & & Socs2 & & 0.001 & 0.031 \\
\hline Hoxb4 & -6.121 & 00 & 0.026 & Sorl1 & -1 & 0.002 & 0.034 \\
\hline Hoxb5 & -6.619 & 000 & 0.020 & Spred2 & -1 & 0.008 & 0.064 \\
\hline Hoxb9 & -9.266 & .000 & 0.024 & Spryd7 & -1 & 0.010 & 0.068 \\
\hline Hoxc6 & -11.922 & 0.000 & 0.024 & Srek1 & & 0.009 & 0.067 \\
\hline Hoxd10 & -11.238 & 0.000 & 0.023 & Ssbp3 & -1 & 0.008 & 0.064 \\
\hline Hoxd4 & -2.142 & 0.003 & 0.046 & St8sia3 & & 0.007 & 0.060 \\
\hline Hoxd8 & -1.603 & 0.004 & 0.048 & Stag2 & -1 & 0.005 & 0.053 \\
\hline Hoxd9 & -3.929 & 0.001 & 0.030 & Sycp3 & -1 & 0.019 & 0.089 \\
\hline Hs6st2 & -2.118 & 0.004 & 0.050 & Synpr & -1.887 & 0.001 & 0.029 \\
\hline Hsp90ab1 & -1.392 & 0.006 & 0.056 & Syt1 & -1 & 0.000 & 0.021 \\
\hline Idh1 & -2.275 & 0.000 & 0.047 & Syt4 & -2 & 0.004 & 0.052 \\
\hline Idi1 & -1.751 & 0.022 & & Sy & & 0.001 & 0.029 \\
\hline Ids & -1.571 & 0.009 & 0.067 & Syt9 & & 0.000 & 0.026 \\
\hline Il10rb & -1.208 & 0.020 & 0.093 & Tac1 & -1.726 & 0.000 & 0.028 \\
\hline II6st & -1.838 & 0.001 & 0.030 & Taf1 & -1.263 & 0.024 & 0.100 \\
\hline Impad1 & -1.490 & 0.002 & 0.035 & Taok1 & -1.691 & 0.005 & 0.055 \\
\hline Ina & -2.171 & 0.000 & 0.028 & $T / x 3$ & -1.349 & 0.001 & 0.030 \\
\hline Irf2 & -1.226 & 0.000 & 0.029 & Tmem158 & -1.431 & 0.003 & 0.045 \\
\hline Izumo4 & -1.366 & 0.009 & 0.068 & Tmem 164 & -1.228 & 0.008 & 0.063 \\
\hline
\end{tabular}

Table 2. Continued

\begin{tabular}{|c|c|c|c|c|c|c|c|}
\hline Genes & $\begin{array}{l}\text { Log2 fold } \\
\text { change }\end{array}$ & $p$ & $q$ & Genes & $\begin{array}{l}\text { Log2 fold } \\
\text { change }\end{array}$ & $p$ & $q$ \\
\hline Kcna4 & -1.408 & 0.010 & 0.069 & Tmem 185b & -1.358 & 0.019 & 0.088 \\
\hline Kcnab1 & -1.296 & 0.003 & 0.046 & Tmem $200 a$ & -1.785 & 0.003 & 0.041 \\
\hline Kcnb2 & -2.196 & 0.001 & 0.032 & Tmem233 & -1.233 & 0.019 & 0.089 \\
\hline Kent1 & -1.215 & 0.017 & 0.086 & Tmem $255 a$ & -1.367 & 0.001 & 0.033 \\
\hline Kdelc2 & -1.279 & 0.010 & 0.070 & Imem56 & -1.466 & 0.009 & 0.068 \\
\hline$K d m 7 a$ & -1.460 & 0.009 & 0.068 & Tmtc2 & -1.914 & 0.015 & 0.082 \\
\hline Kif5b & -1.301 & 0.008 & 0.064 & $\operatorname{Tm} \times 3$ & -1.230 & 0.008 & 0.064 \\
\hline KIf7 & -1.346 & 0.000 & 0.026 & Tnnc2 & -6.667 & 0.001 & 0.030 \\
\hline Larp1 & -1.374 & 0.006 & 0.056 & Tnnt3 & -7.079 & 0.000 & 0.028 \\
\hline Lbh & -1.629 & 0.003 & 0.042 & Тор2a & -1.930 & 0.008 & 0.064 \\
\hline Lcor & -1.431 & 0.008 & 0.063 & $\operatorname{Tra2a}$ & -1.451 & 0.015 & 0.081 \\
\hline$\lfloor d b 2$ & -1.834 & 0.001 & 0.031 & Trp53bp1 & -1.257 & 0.004 & 0.049 \\
\hline Ldlr & -1.708 & 0.002 & 0.034 & $\operatorname{Trpc3}$ & -1.616 & 0.012 & 0.073 \\
\hline Lifr & -1.742 & 0.005 & 0.052 & Trpv1 & -2.016 & 0.008 & 0.065 \\
\hline Lonrf1 & -1.453 & 0.002 & 0.035 & $U b b$ & -1.257 & 0.022 & 0.097 \\
\hline Lox & -2.537 & 0.018 & 0.087 & Ubqln2 & -1.407 & 0.004 & 0.050 \\
\hline Lpar3 & -1.721 & 0.003 & 0.046 & Ugcg & -1.398 & 0.007 & 0.060 \\
\hline Lrfn1 & -1.409 & 0.000 & 0.022 & Unc13c & -1.455 & 0.002 & 0.037 \\
\hline $\operatorname{Lrrc} 8 b$ & -1.402 & 0.001 & 0.030 & Usp17la & -1.946 & 0.011 & 0.072 \\
\hline Lrrtm2 & -1.389 & 0.018 & 0.088 & Usp9x & -1.500 & 0.011 & 0.072 \\
\hline Ly86 & -2.557 & 0.009 & 0.068 & Vwa5a & -1.352 & 0.002 & 0.040 \\
\hline Magi3 & -1.665 & 0.001 & 0.030 & Wasf1 & -1.501 & 0.000 & 0.020 \\
\hline Mal2 & -2.994 & 0.000 & 0.029 & Wfdc2 & -1.246 & 0.019 & 0.090 \\
\hline$M b$ & -6.746 & 0.000 & 0.027 & Xirp2 & -8.656 & 0.000 & 0.026 \\
\hline Mbnl1 & -1.392 & 0.018 & 0.088 & Yod1 & -1.273 & 0.009 & 0.067 \\
\hline Mbnl2 & -1.795 & 0.000 & 0.029 & Zbtb44 & -1.315 & 0.015 & 0.080 \\
\hline Mcoln1 & -1.305 & 0.010 & 0.070 & Zdhhc13 & -1.497 & 0.000 & 0.028 \\
\hline$M f s d 7 b$ & -1.521 & 0.007 & 0.062 & Zeb2 & -1.453 & 0.011 & 0.070 \\
\hline \multirow[t]{2}{*}{ Mon1a } & -1.310 & 0.000 & 0.029 & Zfh $\times 2$ & -1.542 & 0.016 & 0.084 \\
\hline & & & & Zfhx3 & -1.317 & 0.007 & 0.060 \\
\hline
\end{tabular}

2 PCs (PC1 $=$ difference between DRG and TG transcriptomes; and PC2 $=$ difference between the DRG and TG Nav1.8-TRAP translatomes) explained the majority of the variance seen in the dataset (Fig. 3B). Further clustering analysis confirmed the findings of the PC analysis (Fig. 3C).

Analysis of the input transcriptome data between TG and DRG revealed that 379 genes were significantly enriched in the TG and 315 in the DRG (Fig. 4A; Tables 1, 2). Among these 315 genes in the DRG, we observed enrichment of the Hox family transcription factors (Fig. 4A). These genes are well-known regulators of rostral to caudal segmental development, so enrichment in DRG is expected given the rostral-caudal extent of the DRG (Kammermeier and Reichert, 2001). Among the 379 genes enriched in the TG, we found particularly high expression and enrichment of Fth1 and Pak1 (Fig. 4A). Analysis of the Nav1.8TRAP dataset revealed 372 genes enriched in the TG and 348 in the DRG (Fig. 4A; Tables 3, 4). Consistent with the transcriptome results, the Hox genes showed a highly enriched translational profile in the DRG (Fig. 4A). Among the top mRNAs highly associated with ribosomes in the TG Nav1.8-TRAP dataset, we found Nme3, Il1 rl2, and Edf1(Fig. 4A). None of these 3 genes has been associated with a specific TG function previously, although the Illrl2 gene encodes a receptor for interleukin $1 \beta$ (IL1 $\beta)$, which activates TG nociceptors through a mechanism that has previously been attributed to IL1 $\beta$ Type 1 receptors (Takeda et al., 2008). GO term analysis of the differentially expressed genes in the Nav1.8-TRAP datasets revealed an enrichment in specific pathways, including VEGFR, FGFR, as well as the PI3K-mTOR pathway (Fig. 4B). Interestingly, we observed an enrichment in AMP-activated protein kinase (AMPK)-related genes in the 
Table 3. Genes upregulated in the TG Nav1.8-TRAP dataset

\begin{tabular}{|c|c|c|c|c|c|c|c|c|c|c|c|c|c|c|c|}
\hline Genes & $\begin{array}{l}\text { Log2 fold } \\
\text { change }\end{array}$ & $p$ & $q$ & Genes & $\begin{array}{l}\text { Log2 fold } \\
\text { change }\end{array}$ & $p$ & $q$ & Genes & $\begin{array}{l}\text { Log2 fold } \\
\text { change }\end{array}$ & $p$ & $q$ & Genes & $\begin{array}{l}\text { Log2 fold } \\
\text { change }\end{array}$ & $p$ & $q$ \\
\hline 0610009B22Rik & 3.295 & 0.001 & 0.031 & Nap1/5 & 2.348 & 0.001 & 0.029 & Clmp & 2.253 & 0.019 & 0.094 & Rab28 & 2.269 & 0.001 & 0.032 \\
\hline 1110032A03Rik & 2.542 & 0.007 & 0.059 & Nbea & 2.525 & 0.001 & 0.028 & Cnih2 & 4.508 & 0.007 & 0.061 & Rab33a & 3.270 & 0.015 & 0.085 \\
\hline 1110065P20Rik & 5.685 & 0.001 & 0.032 & $\mathrm{Nb} / 1$ & 2.738 & 0.015 & 0.085 & Cnrip1 & 2.211 & 0.008 & 0.066 & Rab35 & 2.067 & 0.012 & 0.078 \\
\hline 1700037C18Rik & 2.913 & 0.010 & 0.072 & Ndel1 & 3.132 & 0.000 & 0.010 & Commd1 & 3.042 & 0.003 & 0.045 & $R a b 4 b$ & 2.707 & 0.008 & 0.065 \\
\hline 2010107E04Rik & 2.008 & 0.000 & 0.016 & Ndufa1 & 2.495 & 0.002 & 0.034 & Commd4 & 2.980 & 0.019 & 0.094 & Rad54l & 5.057 & 0.001 & 0.026 \\
\hline 2210013021Rik & 2.570 & 0.006 & 0.056 & Ndufb2 & 3.065 & 0.002 & 0.034 & $\operatorname{Cog} 3$ & 3.088 & 0.011 & 0.075 & Rad9b & 2.382 & 0.012 & 0.079 \\
\hline 2700094K13Rik & 3.894 & 0.004 & 0.049 & Ndufb5 & 2.214 & 0.001 & 0.027 & Cox6b1 & 2.708 & 0.000 & 0.023 & Rho & 4.096 & 0.003 & 0.040 \\
\hline 9430016H08Rik & 3.239 & 0.016 & 0.089 & Ndufb 9 & 2.915 & 0.000 & 0.017 & Cox7a2 & 2.299 & 0.006 & 0.056 & Rhog & 4.929 & 0.012 & 0.078 \\
\hline Aard & 7.736 & 0.005 & 0.050 & Ndufs2 & 2.315 & 0.003 & 0.043 & Cox7b & 4.295 & 0.000 & 0.017 & Rnase4 & 2.128 & 0.018 & 0.092 \\
\hline Acp1 & 2.522 & 0.004 & 0.049 & Ngfr & 2.166 & 0.011 & 0.075 & $\mathrm{Cox} 7 \mathrm{c}$ & 2.255 & 0.001 & 0.028 & Rnf114 & 3.644 & 0.013 & 0.082 \\
\hline Adam9 & 4.085 & 0.004 & 0.045 & Nme3 & 11.466 & 0.000 & 0.020 & Cox8a & 2.576 & 0.000 & 0.021 & Rnf215 & 4.125 & 0.006 & 0.058 \\
\hline Adra2c & 5.673 & 0.001 & 0.025 & Nrn1l & 4.040 & 0.009 & 0.068 & Crlf2 & 3.905 & 0.011 & 0.075 & Rnf7 & 2.072 & 0.013 & 0.081 \\
\hline Akirin1 & 2.298 & 0.009 & 0.068 & Nsun3 & 3.157 & 0.018 & 0.093 & Crtc2 & 5.091 & 0.001 & 0.023 & Romo1 & 2.456 & 0.000 & 0.021 \\
\hline Akt1 & 3.554 & 0.006 & 0.058 & $\mathrm{Nt} 5 \mathrm{~m}$ & 3.252 & 0.017 & 0.090 & Crtc3 & 3.703 & 0.018 & 0.092 & Rp/10 & 3.076 & 0.013 & 0.080 \\
\hline Alkbh3 & 7.497 & 0.002 & 0.035 & Nubp2 & 5.238 & 0.007 & 0.063 & Ctxn3 & 2.686 & 0.000 & 0.023 & Rpl28 & 2.167 & 0.019 & 0.095 \\
\hline Amacr & 5.927 & 0.013 & 0.082 & Nudt10 & 4.615 & 0.010 & 0.072 & Cyb5a & 2.330 & 0.012 & 0.079 & Rpl29 & 5.333 & 0.021 & 0.099 \\
\hline Anapc13 & 3.962 & 0.015 & 0.087 & Nudt11 & 3.346 & 0.018 & 0.093 & Cyc1 & 3.989 & 0.013 & 0.080 & Rp/35 & 3.039 & 0.003 & 0.040 \\
\hline Ankrd24 & 2.576 & 0.012 & 0.078 & Nudt7 & 4.780 & 0.002 & 0.037 & Cystm1 & 3.446 & 0.002 & 0.038 & Rp/37 & 2.967 & 0.003 & 0.042 \\
\hline Anxa5 & 2.142 & 0.004 & 0.047 & Nup37 & 4.108 & 0.000 & 0.017 & Dad1 & 4.088 & 0.006 & 0.056 & Rp/39 & 3.241 & 0.001 & 0.027 \\
\hline Apip & 7.421 & 0.003 & 0.042 & Ost4 & 2.585 & 0.004 & 0.050 & Dalrd3 & 7.942 & 0.002 & 0.036 & $\operatorname{Rps} 23$ & 2.219 & 0.020 & 0.097 \\
\hline Apln & 4.200 & 0.009 & 0.069 & Ostf1 & 2.376 & 0.003 & 0.042 & Dda1 & 2.393 & 0.017 & 0.091 & $\operatorname{Rps} 29$ & 2.912 & 0.002 & 0.037 \\
\hline Arl5a & 2.178 & 0.002 & 0.038 & Pacsin1 & 2.217 & 0.016 & 0.089 & Dlg2 & 4.233 & 0.010 & 0.072 & Rpusd1 & 3.617 & 0.008 & 0.064 \\
\hline Arl6 & 4.831 & 0.003 & 0.042 & Pak1 & 5.331 & 0.006 & 0.056 & Dnajc12 & 3.352 & 0.016 & 0.088 & Rraga & 2.131 & 0.000 & 0.023 \\
\hline Arl $8 a$ & 2.045 & 0.008 & 0.066 & Pak3 & 3.228 & 0.009 & 0.069 & Dnal4 & 4.100 & 0.012 & 0.076 & Rtn4r & 2.805 & 0.001 & 0.028 \\
\hline Armc1 & 2.080 & 0.016 & 0.089 & Parp3 & 2.863 & 0.010 & 0.072 & Dpep2 & 4.510 & 0.001 & 0.026 & Rxrg & 4.463 & 0.004 & 0.049 \\
\hline Arpc1b & 3.965 & 0.000 & 0.020 & Pcbd2 & 3.529 & 0.002 & 0.037 & Dpm3 & 3.461 & 0.019 & 0.095 & Sac3d1 & 4.162 & 0.009 & 0.070 \\
\hline $\operatorname{Arpc5}$ & 2.223 & 0.002 & 0.039 & Pcolce2 & 3.567 & 0.003 & 0.042 & Dzank1 & 3.857 & 0.011 & 0.074 & Sap18 & 3.653 & 0.019 & 0.095 \\
\hline Asna1 & 3.806 & 0.010 & 0.073 & Pcp4/1 & 3.151 & 0.004 & 0.045 & Edf1 & 2.907 & 0.000 & 0.002 & Sdhb & 4.145 & 0.013 & 0.082 \\
\hline $\operatorname{Atg} 4 \mathrm{C}$ & 4.484 & 0.001 & 0.028 & Pcsk1n & 2.681 & 0.010 & 0.072 & Eef1a1 & 2.126 & 0.016 & 0.088 & Sdhd & 2.350 & 0.008 & 0.066 \\
\hline Atox1 & 2.116 & 0.010 & 0.072 & Pcsk7 & 4.142 & 0.005 & 0.050 & Efcc1 & 5.725 & 0.001 & 0.032 & $\operatorname{Sec} 13$ & 5.366 & 0.002 & 0.037 \\
\hline $\operatorname{Atp5c1}$ & 2.674 & 0.003 & 0.040 & Pdcd6 & 2.770 & 0.009 & 0.067 & Eif4a3 & 2.748 & 0.008 & 0.066 & Sec23ip & 2.817 & 0.014 & 0.083 \\
\hline Atp5d & 2.552 & 0.006 & 0.058 & Pde6d & 5.181 & 0.004 & 0.050 & $E m b$ & 2.251 & 0.000 & 0.017 & Sep15 & 2.455 & 0.009 & 0.068 \\
\hline Atp5g1 & 2.613 & 0.003 & 0.040 & Pdlim2 & 3.067 & 0.001 & 0.027 & Enox1 & 3.300 & 0.017 & 0.090 & Sepp1 & 2.412 & 0.013 & 0.081 \\
\hline Atp5s & 6.255 & 0.000 & 0.018 & Pdzd9 & 2.834 & 0.005 & 0.050 & Epm2a & 2.756 & 0.003 & 0.041 & Serp2 & 3.709 & 0.004 & 0.046 \\
\hline Atp6v0d1 & 3.072 & 0.000 & 0.018 & Pex11b & 7.187 & 0.000 & 0.003 & Esyt1 & 4.806 & 0.004 & 0.045 & Serping1 & 4.031 & 0.007 & 0.060 \\
\hline Avpi1 & 2.049 & 0.005 & 0.053 & Pfdn1 & 2.368 & 0.013 & 0.082 & Exd2 & 5.752 & 0.000 & 0.016 & $\operatorname{sh} 2 d 3 c$ & 3.339 & 0.019 & 0.095 \\
\hline Banf1 & 2.411 & 0.001 & 0.028 & Pfkm & 2.196 & 0.011 & 0.074 & Exosc6 & 4.476 & 0.005 & 0.053 & Sh3bgrl & 2.659 & 0.004 & 0.049 \\
\hline Bbs 9 & 4.103 & 0.021 & 0.099 & Phospho2 & 2.332 & 0.013 & 0.082 & Fabp7 & 3.901 & 0.001 & 0.024 & Sh3bgrl3 & 2.229 & 0.001 & 0.032 \\
\hline Bloc1s1 & 2.178 & 0.015 & 0.085 & Phpt1 & 2.748 & 0.007 & 0.063 & Fam105a & 3.610 & 0.013 & 0.082 & Shd & 8.734 & 0.007 & 0.061 \\
\hline Bloc1s3 & 4.164 & 0.003 & 0.042 & Pigh & 2.609 & 0.011 & 0.075 & Fam188a & 2.065 & 0.011 & 0.075 & Shisa5 & 2.629 & 0.011 & 0.074 \\
\hline Btbd2 & 2.239 & 0.002 & 0.036 & Pin1 & 3.518 & 0.001 & 0.031 & Fam58b & 3.355 & 0.002 & 0.036 & Sirt2 & 3.344 & 0.020 & 0.098 \\
\hline C77080 & 3.683 & 0.000 & 0.016 & Pla2g16 & 2.041 & 0.017 & 0.091 & Fam $89 a$ & 3.170 & 0.010 & 0.072 & Sirt3 & 3.145 & 0.017 & 0.090 \\
\hline Cacng7 & 2.455 & 0.005 & 0.054 & Plekhb1 & 3.646 & 0.014 & 0.083 & Far2 & 3.913 & 0.006 & 0.056 & Sla2 & 2.598 & 0.002 & 0.037 \\
\hline Calca & 2.290 & 0.001 & 0.027 & Plpp1 & 4.280 & 0.017 & 0.091 & Farp1 & 6.925 & 0.000 & 0.016 & Slc22a17 & 3.985 & 0.018 & 0.092 \\
\hline Calm2 & 2.036 & 0.014 & 0.085 & Pole3 & 3.127 & 0.020 & 0.097 & Fbxl16 & 2.675 & 0.007 & 0.060 & Slc $24 a 2$ & 2.209 & 0.001 & 0.028 \\
\hline Camk2g & 2.396 & 0.003 & 0.044 & Polr2d & 6.787 & 0.000 & 0.004 & $\mathrm{Fbx02}$ & 3.859 & 0.007 & 0.063 & Slc25a3 & 3.404 & 0.006 & 0.058 \\
\hline Cbln 1 & 6.228 & 0.004 & 0.047 & Polr2j & 6.438 & 0.001 & 0.027 & $F b \times 027$ & 8.071 & 0.000 & 0.023 & SIc25a43 & 2.557 & 0.019 & 0.095 \\
\hline Ccdc88a & 2.029 & 0.007 & 0.059 & Polr2l & 2.072 & 0.011 & 0.076 & Fgfg & 4.057 & 0.007 & 0.063 & $\operatorname{sic} 35 d 2$ & 6.734 & 0.000 & 0.018 \\
\hline Cdc123 & 2.146 & 0.020 & 0.098 & Polr2m & 2.330 & 0.005 & 0.050 & Fgfr2 & 6.744 & 0.003 & 0.043 & $S / c 3 a 2$ & 3.247 & 0.001 & 0.025 \\
\hline Cdk2ap1 & 4.137 & 0.003 & 0.040 & Pomgnt1 & 3.461 & 0.020 & 0.099 & Fhl1 & 2.487 & 0.001 & 0.024 & Slc45a4 & 2.344 & 0.009 & 0.068 \\
\hline$C d k n 1 b$ & 2.713 & 0.001 & 0.024 & Ppm1j & 2.163 & 0.009 & 0.067 & Fkbp2 & 2.064 & 0.008 & 0.065 & Slc46a3 & 5.451 & 0.003 & 0.040 \\
\hline Cdr2l & 2.794 & 0.007 & 0.061 & Ppp2r5c & 2.205 & 0.014 & 0.084 & Fsd1 & 2.330 & 0.010 & 0.072 & Slc6a15 & 3.041 & 0.014 & 0.084 \\
\hline Cebpzos & 3.122 & 0.004 & 0.049 & Praf2 & 2.266 & 0.019 & 0.096 & Fth1 & 3.259 & 0.001 & 0.023 & Slco2b1 & 5.287 & 0.004 & 0.047 \\
\hline Cela1 & 4.351 & 0.004 & 0.049 & Prkcd & 2.054 & 0.003 & 0.040 & Fxyd6 & 2.042 & 0.006 & 0.056 & Slitrk1 & 3.031 & 0.018 & 0.094 \\
\hline Cenpq & 4.971 & 0.002 & 0.037 & Prkcdbp & 6.444 & 0.002 & 0.035 & Gabrg1 & 3.806 & 0.000 & 0.018 & Smdt1 & 2.724 & 0.000 & 0.019 \\
\hline Cfap69 & 3.913 & 0.012 & 0.077 & Prkrir & 2.325 & 0.008 & 0.064 & Galnt18 & 2.412 & 0.013 & 0.080 & Smim 12 & 2.038 & 0.000 & 0.021 \\
\hline Cfl1 & 2.008 & 0.001 & 0.032 & Prorsd1 & 5.144 & 0.008 & 0.065 & Gatad1 & 2.512 & 0.004 & 0.047 & Smim8 & 2.292 & 0.003 & 0.042 \\
\hline Chchd1 & 3.792 & 0.000 & 0.017 & Psma1 & 2.561 & 0.017 & 0.091 & Gipc1 & 3.901 & 0.018 & 0.093 & Snapc5 & 2.360 & 0.016 & 0.088 \\
\hline Chchd10 & 3.927 & 0.011 & 0.074 & Psmb11 & 2.717 & 0.000 & 0.021 & Glb1/2 & 4.152 & 0.008 & 0.066 & Snn & 3.703 & 0.015 & 0.085 \\
\hline Chchd4 & 2.146 & 0.003 & 0.040 & Psmc3ip & 6.150 & 0.007 & 0.061 & Gm15440 & 5.964 & 0.012 & 0.079 & Snrpb & 2.974 & 0.002 & 0.037 \\
\hline Chd3os & 2.704 & 0.003 & 0.040 & Qars & 2.763 & 0.012 & 0.079 & Gm5113 & 6.649 & 0.001 & 0.032 & Snrpn & 3.974 & 0.002 & 0.037 \\
\hline Chmp6 & 2.299 & 0.021 & 0.099 & Rab10 & 2.106 & 0.010 & 0.071 & Gmnn & 4.878 & 0.001 & 0.026 & $\operatorname{Snx} 3$ & 2.491 & 0.002 & 0.037 \\
\hline Chodl & 5.030 & 0.016 & 0.089 & Rab15 & 3.719 & 0.013 & 0.080 & Gng5 & 4.458 & 0.000 & 0.018 & Spcs1 & 2.441 & 0.021 & 0.099 \\
\hline Chp1 & 4.425 & 0.000 & 0.017 & Rab1a & 4.034 & 0.008 & 0.064 & Gng8 & 4.700 & 0.006 & 0.057 & Spon1 & 3.949 & 0.021 & 0.099 \\
\hline
\end{tabular}

Table 3. Continued 
Table 3. Continued

\begin{tabular}{|c|c|c|c|c|c|c|c|}
\hline Genes & $\begin{array}{l}\text { Log2 fold } \\
\text { change }\end{array}$ & $p$ & $q$ & Genes & $\begin{array}{l}\text { Log2 fold } \\
\text { change }\end{array}$ & $p$ & $q$ \\
\hline Golga1 & 3.883 & 0.016 & 0.089 & Srp14 & 2.998 & 0.000 & 0.021 \\
\hline Gpr35 & 3.338 & 0.017 & 0.091 & Ssr4 & 3.347 & 0.011 & 0.075 \\
\hline Gramd1b & 2.251 & 0.000 & 0.020 & Stau2 & 2.158 & 0.008 & 0.066 \\
\hline Grpel2 & 3.678 & 0.018 & 0.092 & Strada & 5.449 & 0.008 & 0.064 \\
\hline Gtf2i & 3.014 & 0.011 & 0.075 & Stx2 & 3.170 & 0.000 & 0.017 \\
\hline H2-T23 & 4.183 & 0.010 & 0.070 & Suclg1 & 2.738 & 0.010 & 0.073 \\
\hline$H 2 a f z$ & 2.207 & 0.003 & 0.042 & Supt $4 a$ & 3.527 & 0.001 & 0.032 \\
\hline Hist $3 h 2 b a$ & 2.569 & 0.016 & 0.089 & Tagln3 & 3.422 & 0.004 & 0.047 \\
\hline Hsbp1 & 2.142 & 0.016 & 0.089 & Tbc1d14 & 2.192 & 0.009 & 0.070 \\
\hline$I d h 3 b$ & 3.457 & 0.001 & 0.025 & $\mathrm{Tfb} 1 \mathrm{~m}$ & 3.745 & 0.013 & 0.082 \\
\hline Ift122 & 3.334 & 0.011 & 0.074 & Thoc7 & 2.488 & 0.008 & 0.066 \\
\hline Ift20 & 2.144 & 0.021 & 0.099 & Tifab & 5.602 & 0.004 & 0.045 \\
\hline Ift43 & 6.053 & 0.018 & 0.094 & Timm8b & 4.092 & 0.007 & 0.061 \\
\hline Igsf21 & 4.754 & 0.014 & 0.084 & Tjap1 & 3.096 & 0.021 & 0.099 \\
\hline$\| 11 r l 2$ & 8.506 & 0.000 & 0.016 & Tmem106b & 2.653 & 0.011 & 0.076 \\
\hline Imp3 & 2.343 & 0.001 & 0.032 & Tmem199 & 4.071 & 0.014 & 0.084 \\
\hline Inpp5j & 4.481 & 0.008 & 0.064 & Tmem216 & 8.212 & 0.001 & 0.026 \\
\hline Isca2 & 2.096 & 0.011 & 0.074 & Tmem230 & 2.058 & 0.006 & 0.058 \\
\hline Iscu & 3.142 & 0.006 & 0.058 & Tmem258 & 5.441 & 0.000 & 0.017 \\
\hline Kenip1 & 5.122 & 0.001 & 0.032 & Tmem53 & 3.911 & 0.009 & 0.069 \\
\hline Kcnip4 & 2.177 & 0.002 & 0.038 & Tmem62 & 4.157 & 0.014 & 0.083 \\
\hline Kctd8 & 2.176 & 0.006 & 0.055 & Tnfrsfia & 5.108 & 0.008 & 0.065 \\
\hline KIf8 & 4.565 & 0.004 & 0.047 & Tnfsfm13 & 5.814 & 0.015 & 0.085 \\
\hline Lamtor5 & 2.577 & 0.005 & 0.055 & Tomm7 & 2.169 & 0.000 & 0.017 \\
\hline Lcmt1 & 2.326 & 0.006 & 0.056 & Tpd52/1 & 4.373 & 0.009 & 0.068 \\
\hline Lgals3bp & 6.428 & 0.008 & 0.064 & Tpgs2 & 3.087 & 0.004 & 0.049 \\
\hline Limk1 & 2.887 & 0.002 & 0.034 & Трpр3 & 2.673 & 0.000 & 0.015 \\
\hline Lipa & 2.672 & 0.005 & 0.054 & Trappc1 & 3.056 & 0.019 & 0.095 \\
\hline Lix1 & 2.853 & 0.002 & 0.035 & Trdmt1 & 5.930 & 0.000 & 0.021 \\
\hline Lrrc8a & 2.077 & 0.000 & 0.018 & Triap1 & 2.123 & 0.008 & 0.064 \\
\hline$L t b r$ & 3.603 & 0.007 & 0.063 & Trim $12 a$ & 2.989 & 0.011 & 0.075 \\
\hline$L x n$ & 2.922 & 0.001 & 0.026 & Trim9 & 3.505 & 0.014 & 0.084 \\
\hline Lyrm2 & 4.541 & 0.000 & 0.023 & Trnp1 & 2.255 & 0.003 & 0.040 \\
\hline Lyrm5 & 2.559 & 0.019 & 0.095 & Tspan17 & 5.060 & 0.008 & 0.066 \\
\hline M6pr & 2.171 & 0.007 & 0.062 & Tspan3 & 2.352 & 0.003 & 0.040 \\
\hline Manbal & 2.620 & 0.009 & 0.069 & Tspan7 & 2.270 & 0.001 & 0.027 \\
\hline Map1/c3b & 2.978 & 0.001 & 0.028 & $T u b$ & 6.954 & 0.000 & 0.016 \\
\hline Mapkap1 & 2.645 & 0.010 & 0.070 & $T u b b 4 b$ & 2.426 & 0.002 & 0.037 \\
\hline Mapkapk5 & 2.476 & 0.004 & 0.045 & Tyro3 & 5.176 & 0.000 & 0.023 \\
\hline Mast2 & 4.666 & 0.004 & 0.049 & Ubald1 & 3.325 & 0.003 & 0.040 \\
\hline$M b d 2$ & 3.693 & 0.012 & 0.078 & Ubl5 & 2.981 & 0.006 & 0.058 \\
\hline Mblac2 & 3.198 & 0.007 & 0.063 & Ulk1 & 2.369 & 0.009 & 0.067 \\
\hline Mboat7 & 4.459 & 0.005 & 0.055 & Uqcrb & 3.669 & 0.001 & 0.025 \\
\hline Mgst3 & 5.093 & 0.004 & 0.046 & Uqcrfs1 & 2.994 & 0.005 & 0.052 \\
\hline Mid2 & 6.101 & 0.001 & 0.028 & Usmg5 & 3.313 & 0.001 & 0.027 \\
\hline Minpp1 & 4.786 & 0.004 & 0.047 & Vkorc1l1 & 3.000 & 0.010 & 0.072 \\
\hline Mipol1 & 3.394 & 0.015 & 0.086 & Vta1 & 2.955 & 0.012 & 0.076 \\
\hline Mlf2 & 2.377 & 0.000 & 0.018 & Vwc2l & 3.597 & 0.010 & 0.071 \\
\hline Mob3b & 3.803 & 0.020 & 0.099 & Wbp1 & 4.041 & 0.019 & 0.095 \\
\hline Mrp/18 & 2.783 & 0.000 & 0.023 & Wbp2 & 2.195 & 0.000 & 0.002 \\
\hline Mrpl27 & 2.877 & 0.013 & 0.081 & Wdr59 & 3.560 & 0.004 & 0.049 \\
\hline Mrps14 & 4.314 & 0.004 & 0.049 & Wfs1 & 4.090 & 0.009 & 0.067 \\
\hline Mrps36 & 4.605 & 0.006 & 0.058 & Wisp1 & 5.770 & 0.008 & 0.064 \\
\hline Msra & 3.657 & 0.013 & 0.082 & Ypel3 & 2.511 & 0.003 & 0.042 \\
\hline$m t-N d 2$ & 2.268 & 0.007 & 0.063 & Ywhaq & 2.191 & 0.001 & 0.026 \\
\hline$M x d 3$ & 2.495 & 0.007 & 0.060 & Zdhhc6 & 4.565 & 0.008 & 0.064 \\
\hline Mzt1 & 2.130 & 0.000 & 0.021 & Zfp932 & 3.625 & 0.020 & 0.097 \\
\hline Naa38 & 4.005 & 0.018 & 0.093 & Zfp944 & 6.108 & 0.006 & 0.056 \\
\hline
\end{tabular}

DRG-TRAP dataset (Fig. 4B). This finding is intriguing because the AMPK pathway is a negative regulator of PI3K-mTOR signaling (Hardie, 2014, 2015) and suggests shifting in the balance between these two signaling pathways between the DRG and TG.
Table 4. Genes upregulated in the DRG Nav1.8-TRAP dataset

\begin{tabular}{|c|c|c|c|c|c|c|c|}
\hline Genes & $\begin{array}{l}\text { Log2 fold } \\
\text { change }\end{array}$ & $p$ & $q$ & Genes & $\begin{array}{l}\text { Log2 fold } \\
\text { change }\end{array}$ & $p$ & $q$ \\
\hline 2810417H13Rik & -7.030 & 0.002 & 0.036 & Mif & -2.343 & 0.012 & 0.078 \\
\hline A430078G23Rik & -4.575 & 0.005 & 0.055 & Mmp15 & -2.137 & 0.005 & 0.055 \\
\hline Abca6 & -10.621 & 0.000 & 0.017 & Mon1a & -2.894 & 0.002 & 0.037 \\
\hline Abhd17c & -2.949 & 0.000 & 0.017 & Mrpl14 & -3.067 & 0.020 & 0.099 \\
\hline$A b t 1$ & -2.637 & 0.008 & 0.065 & Mrpl37 & -2.040 & 0.003 & 0.044 \\
\hline Acaca & -2.654 & 0.002 & 0.037 & Mrto4 & -3.186 & 0.006 & 0.056 \\
\hline Acacb & -3.534 & 0.005 & 0.055 & Msn & -2.617 & 0.003 & 0.040 \\
\hline Acot6 & -7.991 & 0.001 & 0.029 & $M t-C 03$ & -6.804 & 0.001 & 0.026 \\
\hline Acta1 & -5.881 & 0.006 & 0.057 & Mterf4 & -2.725 & 0.009 & 0.068 \\
\hline Actb & -2.404 & 0.000 & 0.016 & Mvd & -2.888 & 0.002 & 0.035 \\
\hline Adap1 & -2.420 & 0.010 & 0.073 & Myc & -6.466 & 0.001 & 0.030 \\
\hline Adgrb3 & -4.063 & 0.019 & 0.095 & Myh1 & -10.133 & 0.002 & 0.035 \\
\hline Adra2a & -8.313 & 0.000 & 0.017 & Myh9 & -2.359 & 0.004 & 0.046 \\
\hline Akap5 & -8.506 & 0.000 & 0.017 & Myo10 & -4.730 & 0.006 & 0.056 \\
\hline Akr7a5 & -2.071 & 0.013 & 0.081 & Myoc & -3.234 & 0.015 & 0.085 \\
\hline Akt1s1 & -2.002 & 0.004 & 0.050 & Nat9 & -3.387 & 0.012 & 0.078 \\
\hline Anapc5 & -2.274 & 0.001 & 0.023 & Ndfip1 & -4.237 & 0.010 & 0.071 \\
\hline Ankrd13d & -2.118 & 0.006 & 0.058 & Ndst3 & -4.583 & 0.007 & 0.062 \\
\hline Anp32b & -2.272 & 0.003 & 0.040 & Ndufa3 & -2.237 & 0.017 & 0.090 \\
\hline Ap1s1 & -2.586 & 0.003 & 0.040 & Ndufs5 & -2.374 & 0.018 & 0.092 \\
\hline Ap2s1 & -2.371 & 0.012 & 0.078 & Ndufs6 & -3.439 & 0.000 & 0.018 \\
\hline Aqr & -2.439 & 0.019 & 0.094 & Nes & -5.213 & 0.001 & 0.030 \\
\hline Arhgap26 & -3.451 & 0.000 & 0.017 & Neurl4 & -2.326 & 0.005 & 0.052 \\
\hline Arhgap39 & -2.113 & 0.006 & 0.058 & Nfkbil1 & -4.943 & 0.001 & 0.027 \\
\hline Arhgef11 & -2.432 & 0.016 & 0.088 & Nfya & -4.346 & 0.018 & 0.093 \\
\hline Ascc2 & -10.550 & 0.001 & 0.024 & Noct & -2.815 & 0.013 & 0.081 \\
\hline Astn2 & -8.207 & 0.001 & 0.027 & Nol10 & -2.001 & 0.001 & 0.024 \\
\hline Atf6b & -2.528 & 0.010 & 0.072 & Nop16 & -2.605 & 0.001 & 0.032 \\
\hline Atg2a & -2.559 & 0.001 & 0.028 & Nptx1 & -2.018 & 0.005 & 0.055 \\
\hline Atm & -2.175 & 0.017 & 0.090 & Npy1r & -3.647 & 0.007 & 0.061 \\
\hline Atp2b4 & -2.641 & 0.000 & 0.017 & Nsun2 & -2.052 & 0.002 & 0.037 \\
\hline Atp6v0a1 & -3.155 & 0.001 & 0.032 & Nts & -6.880 & 0.003 & 0.040 \\
\hline B3gnt8 & -2.129 & 0.015 & 0.085 & Nuak1 & -2.011 & 0.003 & 0.040 \\
\hline Bach1 & -4.987 & 0.002 & 0.035 & Nup155 & -5.434 & 0.000 & 0.021 \\
\hline Baz1b & -2.451 & 0.016 & 0.089 & Nup88 & -2.178 & 0.005 & 0.050 \\
\hline C130074G19Rik & -6.277 & 0.001 & 0.027 & Nyap1 & -2.210 & 0.003 & 0.045 \\
\hline Cadm4 & -2.525 & 0.005 & 0.050 & Obfc1 & -5.064 & 0.003 & 0.041 \\
\hline Capn1 & -2.714 & 0.001 & 0.028 & $0 b 0 \times 3$ & -3.209 & 0.002 & 0.035 \\
\hline Cast & -2.300 & 0.000 & 0.017 & Ogfr & -3.157 & 0.004 & 0.047 \\
\hline Ccdc130 & -5.043 & 0.003 & 0.040 & P2rx3 & -2.594 & 0.015 & 0.086 \\
\hline $\operatorname{Ccdc} 3$ & -2.703 & 0.021 & 0.099 & Pabpn1 & -2.231 & 0.008 & 0.065 \\
\hline$C+t_{5}$ & -2.433 & 0.002 & 0.037 & Palm3 & -2.239 & 0.010 & 0.072 \\
\hline$C c t 7$ & -2.291 & 0.004 & 0.045 & Panx1 & -2.446 & 0.006 & 0.056 \\
\hline Cct8 & -4.490 & 0.002 & 0.034 & Pcbp2 & -5.332 & 0.003 & 0.041 \\
\hline$C d c 26$ & -2.164 & 0.002 & 0.039 & Pcdh11x & -7.446 & 0.000 & 0.003 \\
\hline Cdh1 & -2.230 & 0.004 & 0.049 & Pex6 & -2.364 & 0.005 & 0.055 \\
\hline$C d k 11 b$ & -3.476 & 0.004 & 0.047 & Pfdn2 & -2.988 & 0.004 & 0.045 \\
\hline Cep85I & -9.647 & 0.001 & 0.026 & Pfkp & -2.031 & 0.001 & 0.032 \\
\hline Cetn2 & -2.177 & 0.010 & 0.072 & Pfn1 & -2.932 & 0.000 & 0.020 \\
\hline$C k b$ & -2.670 & 0.002 & 0.035 & Pgls & -2.444 & 0.009 & 0.068 \\
\hline $\mathrm{Ckm}$ & -8.225 & 0.006 & 0.056 & Phldb2 & -8.699 & 0.004 & 0.046 \\
\hline Clgn & -2.261 & 0.000 & 0.017 & Pih1d1 & -2.509 & 0.002 & 0.035 \\
\hline Clint1 & -2.048 & 0.009 & 0.067 & Plcb3 & -3.864 & 0.000 & 0.018 \\
\hline Clip2 & -4.567 & 0.000 & 0.015 & Plec & -3.238 & 0.000 & 0.017 \\
\hline Cln6 & -3.765 & 0.001 & 0.025 & Plekhm1 & -4.182 & 0.005 & 0.053 \\
\hline Col1a1 & -2.067 & 0.011 & 0.074 & Pnpo & -3.077 & 0.008 & 0.066 \\
\hline Col5a2 & -7.121 & 0.021 & 0.100 & Poll & -4.050 & 0.002 & 0.035 \\
\hline Col5a3 & -5.712 & 0.005 & 0.050 & Polr2h & -3.280 & 0.011 & 0.075 \\
\hline Col8a1 & -7.657 & 0.002 & 0.037 & Pop1 & -7.207 & 0.000 & 0.001 \\
\hline Cops6 & -2.017 & 0.002 & 0.037 & Prcc & -2.257 & 0.002 & 0.033 \\
\hline $\operatorname{Cog} 7$ & -2.086 & 0.015 & 0.085 & Prg2 & -10.331 & 0.000 & 0.020 \\
\hline Cpe & -2.053 & 0.002 & 0.034 & Prkag2 & -2.591 & 0.000 & 0.019 \\
\hline Cpt1c & -2.190 & 0.006 & 0.059 & Prrx1 & -5.132 & 0.004 & 0.047 \\
\hline Cpxm1 & -3.325 & 0.005 & 0.052 & Psmb3 & -2.915 & $\begin{array}{c}0.002 \\
\text { (Table co }\end{array}$ & $\begin{array}{r}0.036 \\
\text { tinues) }\end{array}$ \\
\hline
\end{tabular}


Table 4. Continued

\begin{tabular}{|c|c|c|c|c|c|c|c|}
\hline Genes & $\begin{array}{l}\text { Log2 fold } \\
\text { change }\end{array}$ & $p$ & $q$ & Genes & $\begin{array}{l}\text { Log2 fold } \\
\text { change }\end{array}$ & $p$ & $q$ \\
\hline Csgalnact1 & -9.636 & 0.001 & 0.028 & Psmb5 & -2.213 & 0.002 & 0.036 \\
\hline Csnk2a2 & -2.122 & 0.006 & 0.059 & Psmc1 & -2.405 & 0.000 & 0.016 \\
\hline Csrnp1 & -10.669 & 0.001 & 0.030 & Psmd13 & -2.670 & 0.003 & 0.044 \\
\hline Ctu2 & -3.003 & 0.014 & 0.084 & Psmd2 & -2.182 & 0.001 & 0.027 \\
\hline Cul7 & -3.621 & 0.007 & 0.062 & Psmd4 & -2.809 & 0.003 & 0.044 \\
\hline Cwc25 & -2.930 & 0.004 & 0.045 & Ptger1 & -3.477 & 0.018 & 0.094 \\
\hline Dapk2 & -2.224 & 0.014 & 0.084 & Ptms & -3.871 & 0.002 & 0.037 \\
\hline$D d \times 56$ & -2.158 & 0.004 & 0.045 & Ptpn23 & -7.273 & 0.002 & 0.036 \\
\hline Dgkz & -2.686 & 0.003 & 0.040 & Ptprb & -7.624 & 0.000 & 0.004 \\
\hline Disp1 & -2.321 & 0.015 & 0.085 & Ptrf & -4.042 & 0.005 & 0.053 \\
\hline$D \lg 3$ & -3.824 & 0.008 & 0.064 & Pycr2 & -2.066 & 0.015 & 0.085 \\
\hline Dnaja2 & -2.167 & 0.001 & 0.028 & Pygl & -2.181 & 0.014 & 0.083 \\
\hline Dnm1 & -2.469 & 0.009 & 0.068 & Pygo2 & -2.586 & 0.007 & 0.063 \\
\hline Dph2 & -6.419 & 0.000 & 0.017 & Qsox1 & -2.054 & 0.003 & 0.040 \\
\hline Dph7 & -6.003 & 0.000 & 0.014 & R3hdm4 & -2.054 & 0.013 & 0.082 \\
\hline Dpp7 & -2.645 & 0.013 & .080 & Rack1 & -2.006 & 0.005 & 0.055 \\
\hline Dpp8 & -2.446 & 0.003 & 0.042 & Rae1 & -2.346 & 14 & 0.084 \\
\hline Dpy19/4 & -6.024 & 0.001 & 0.028 & Rara & -4.005 & 05 & 0.053 \\
\hline Dpys/5 & -2.386 & 0.016 & 088 & Rbm14 & -6.125 & .001 & 0.032 \\
\hline Drg2 & -2.986 & 0.011 & 0.076 & Rbm6 & -2.191 & 0.007 & 0.061 \\
\hline Ebpl & -2.024 & 0.006 & 0.056 & $R \subset c 2$ & -2.150 & 0.002 & 0.037 \\
\hline Edc4 & -3.342 & 0.003 & 0.040 & Reps1 & -4.535 & 0.000 & 0.022 \\
\hline Ehmt2 & -2.944 & 0.008 & 66 & Rexo4 & -3.169 & 15 & 0.087 \\
\hline Eif2b4 & -2.226 & 0.009 & & $R f c 2$ & -2.711 & 15 & 0.054 \\
\hline Eif2b5 & -2.424 & 0.002 & 0.033 & Riok1 & -2.211 & 17 & 0.090 \\
\hline Eif3j1 & -2.155 & 0.018 & 0.092 & Rnf122 & -2.237 & 0.000 & 0.018 \\
\hline Eif3m & -2.731 & 0.006 & 0.057 & Rnf2 & -2.259 & 0.015 & 0.086 \\
\hline Eif5b & -2.048 & 0.008 & 066 & Rpia & -2.151 & 0.017 & 0.090 \\
\hline Emc1 & -2.455 & 0.004 & 047 & $R p / 24$ & -3.600 & 3 & 0.040 \\
\hline Eps8 & -7.159 & 0.003 & 0.044 & Rplp2 & -2.166 & 03 & 0.040 \\
\hline Fabp4 & -7.365 & 0.000 & 21 & Rrp1 & -2.684 & 0.001 & 0.025 \\
\hline Fam195b & -2 & 0.003 & 0 & Rrp7a & -2.173 & 0.010 & 0.070 \\
\hline Fam 21 & -2.569 & 0.001 & 0.024 & Rsph9 & -3.818 & 0.009 & 0.067 \\
\hline Fam65b & -7.543 & 0.003 & 0.044 & Rsrc1 & -2.223 & 0.005 & 0.054 \\
\hline Fbn1 & -5.976 & 0.004 & & Ryr1 & -9.068 & 0.021 & 0.09 \\
\hline Fh1 & -2.129 & 0.006 & & 51000 & -7.163 & 01 & 0.025 \\
\hline Fh/3 & -3.273 & 0. & 0 & & -6.621 & 0.002 & 0.033 \\
\hline Fkbp10 & -8.824 & 0.001 & 17 & Sae1 & -2.758 & 0.008 & 0.064 \\
\hline Fkbp 14 & -2.261 & 0.005 & 0.053 & Sart3 & -2.174 & 0.001 & 0.024 \\
\hline Fnbp4 & -4.246 & 0.012 & 0.078 & Sass6 & -2.773 & 0.015 & 0.087 \\
\hline Frg1 & -2.094 & 0.001 & 0.030 & $S c n 2 a 1$ & -4.227 & 0.008 & 0.066 \\
\hline Ftsj3 & -2.299 & 0004 & & Sdad1 & -2.183 & 4 & 0.08 \\
\hline Gab2 & -2.044 & 0. & 7 & $S d k 2$ & -4.549 & 0.013 & 0.08 \\
\hline Gdap1/1 & -2.493 & 0.011 & 0.074 & $S d s I$ & -4.243 & 0.017 & 0.092 \\
\hline Gfod2 & -2.657 & 0.010 & 0.071 & Selenbp1 & -8.748 & 0.001 & 0.027 \\
\hline Gga1 & -2.391 & 0.004 & 0.049 & Senp1 & -5.212 & 0.018 & 0.092 \\
\hline Gm21967 & -3.888 & 0.010 & 0.073 & Sept6 & -2.159 & 0.006 & 0.05 \\
\hline Gm42417 & -9.870 & 0.005 & 0.053 & Serpind & -3.644 & 0.014 & 0.0 \\
\hline Golga2 & -2.272 & & & Sertad1 & -2.157 & 0.006 & 0.05 \\
\hline Golga7b & -2.577 & 0.004 & 0.047 & Sfrp5 & -2.379 & 0.001 & 0.02 \\
\hline Gpibb & -2.047 & 0.001 & 0.030 & $\sin 3 b$ & -2.401 & 0.002 & 0.032 \\
\hline Gpr179 & -8.139 & 0.001 & 0.028 & Slc16a3 & -10.302 & 0.005 & 0.050 \\
\hline Gpx4 & -2.608 & 0.009 & 0.069 & SIC25a24 & -8.902 & 0.000 & 0.017 \\
\hline Grce10 & -2.915 & 0.003 & 0.040 & SIC27a4 & -2.479 & 0.020 & 0.0 \\
\hline Gsn & -2.133 & & & Slc39a6 & -2.296 & & 0.036 \\
\hline Gtf2f2 & -9.634 & 0.003 & 0.042 & SIc43a1 & -4.289 & 0.009 & 0.06 \\
\hline Gys1 & -2.353 & 0.000 & 0.016 & Slc51a & -6.814 & 0.000 & 0.01 \\
\hline H2afyz & -3.646 & 0.001 & 0.026 & SIC7a3 & -2.557 & 0.018 & 0.093 \\
\hline$H b a-a 2$ & -3.246 & 0.010 & 0.072 & SIC7a5 & -3.490 & 0.000 & 0.02 \\
\hline Hddc2 & -2.355 & 0.005 & 0.055 & Slfn2 & -8.252 & 0.014 & 0.0 \\
\hline Hdgfrp2 & -2.558 & 0.001 & 0.028 & Smarcd2 & -3.411 & 0.018 & 0.0 \\
\hline$H g f$ & -3.196 & 0.008 & 0.065 & Smarce1 & -2.804 & 0.016 & 0.089 \\
\hline Hmgd & -2.512 & 0.006 & 0.058 & Snf8 & -2.088 & 0.006 & 0.056 \\
\hline Hnrnpf & -2.067 & 0.005 & 0.053 & Snrnp200 & -2.004 & 0.018 & 0.093 \\
\hline
\end{tabular}

Table 4. Continued

\begin{tabular}{|c|c|c|c|c|c|c|c|}
\hline Genes & $\begin{array}{l}\text { Log2 fold } \\
\text { change }\end{array}$ & $p$ & $q$ & Genes & $\begin{array}{l}\text { Log2 fold } \\
\text { change }\end{array}$ & $p$ & $q$ \\
\hline Hnrnpk & -2.677 & 0.000 & 0.016 & Snw1 & -2.689 & 0.000 & 0.016 \\
\hline Hnrnpm & -2.317 & 0.006 & 0.057 & Snx9 & -7.202 & 0.001 & 0.029 \\
\hline Hoxa10 & -7.037 & 0.000 & 0.018 & Sox8 & -8.131 & 0.000 & 0.012 \\
\hline Hoxa7 & -6.596 & 0.000 & 0.018 & Sppl3 & -2.328 & 0.012 & 0.079 \\
\hline Hoxa9 & -6.957 & 0.000 & 0.023 & Spred3 & -4.439 & 0.003 & 0.040 \\
\hline Hoxb2 & -5.943 & 0.000 & 0.019 & Srcap & -3.676 & 0.007 & 0.060 \\
\hline Hoxb4 & -8.161 & 0.000 & 0.017 & Ssb & -2.140 & 0.005 & 0.053 \\
\hline Hoxb5 & -7.221 & 0.000 & 0.017 & Supt16 & -2.360 & 0.000 & 0.018 \\
\hline Hoxb9 & -9.174 & 0.000 & 0.016 & Syp & -2.076 & 0.015 & 0.087 \\
\hline Hoxc6 & -9.853 & 0.000 & 0.002 & Taf $4 b$ & -10.301 & 0.002 & 0.037 \\
\hline Hoxd10 & -7.982 & 0.001 & 0.030 & Tango6 & -10.199 & 0.002 & 0.037 \\
\hline Hoxd4 & -4.810 & 0.001 & 0.026 & Tarbp2 & -3.521 & 0.003 & 0.040 \\
\hline Hoxd8 & -5.752 & 0.000 & 0.020 & Tbc1d10b & -2.056 & 0.011 & 0.074 \\
\hline Hoxd9 & -7.597 & 0.005 & 0.053 & Thap4 & -6.562 & 0.000 & 0.017 \\
\hline Hps4 & -5.021 & 0.003 & 0.042 & Timm50 & -2.701 & 0.011 & 0.074 \\
\hline Hsp90ab1 & -2.714 & 0.000 & 0.018 & $T k t$ & -3.256 & 0.010 & 0.072 \\
\hline Hspa9 & -2.531 & 0.000 & 0.023 & $T / x 3$ & -2.618 & 0.002 & 0.035 \\
\hline ler5l & -5.059 & 0.006 & 0.057 & Tmem 101 & -2.205 & 0.015 & 0.087 \\
\hline Il11ra1 & -2.334 & 0.012 & 0.078 & Tmem 201 & -5.403 & 0.000 & 0.020 \\
\hline Ino80 & -4.042 & 0.006 & 0.057 & Tmem205 & -4.532 & 0.001 & 0.026 \\
\hline Irf5 & -8.535 & 0.000 & 0.004 & Tnnc2 & -9.833 & 0.001 & 0.030 \\
\hline $\operatorname{lrgq}$ & -2.836 & 0.010 & 0.072 & Tnnt3 & -7.545 & 0.002 & 0.038 \\
\hline$K d m 1 a$ & -2.263 & 0.006 & 0.058 & Tnpo2 & -2.492 & 0.007 & 0.063 \\
\hline$K d m 4 b$ & -8.283 & 0.010 & 0.073 & Tnrc18 & -2.271 & 0.005 & 0.053 \\
\hline Kif3a & -2.029 & 0.014 & 0.084 & Top2a & -7.193 & 0.019 & 0.095 \\
\hline Kif3c & -4.039 & 0.002 & 0.035 & Ttbk1 & -2.591 & 0.007 & 0.061 \\
\hline KIf2 & -6.811 & 0.001 & 0.025 & $T u b b 3$ & -2.963 & 0.000 & 0.015 \\
\hline Lcmt2 & -4.928 & 0.003 & 0.040 & Txnrd2 & -2.946 & 0.008 & 0.066 \\
\hline$L d / r$ & -2.419 & 0.001 & 0.024 & $U b c$ & -2.316 & 0.002 & 0.037 \\
\hline Leo1 & -2.433 & 0.008 & 0.065 & Ube2s & -2.379 & 0.009 & 0.069 \\
\hline Lig1 & -2.253 & 0.002 & 0.034 & Uchl1 & -3.022 & 0.009 & 0.069 \\
\hline Loxl1 & -6.618 & 0.001 & 0.030 & Uck2 & -2.829 & 0.006 & 0.056 \\
\hline Lrrc17 & -3.531 & 0.008 & 0.064 & Upf3b & -3.615 & 0.007 & 0.060 \\
\hline Lrrfip1 & -3.487 & 0.005 & 0.050 & Urgcp & -4.220 & 0.001 & 0.029 \\
\hline Lta $4 \mathrm{~h}$ & -2.258 & 0.003 & 0.042 & Usp17la & -7.689 & 0.000 & 0.016 \\
\hline Ltbp3 & -2.457 & 0.007 & 0.062 & Utp18 & -4.338 & 0.009 & 0.068 \\
\hline $\operatorname{Man} 2 b 2$ & -8.690 & 0.000 & 0.018 & Vezt & -2.143 & 0.015 & 0.087 \\
\hline Map1a & -2.487 & 0.002 & 0.035 & Vps33a & -2.623 & 0.008 & 0.066 \\
\hline$M b$ & -9.449 & 0.002 & 0.035 & Vps8 & -3.249 & 0.000 & 0.018 \\
\hline Mboat1 & -5.624 & 0.003 & 0.041 & Xirp2 & -8.750 & 0.002 & 0.038 \\
\hline Mcoln1 & -2.973 & 0.012 & 0.079 & Yipf1 & -2.497 & 0.001 & 0.025 \\
\hline Mdfic & -10.177 & 0.001 & 0.032 & Zfp 212 & -2.281 & 0.013 & 0.081 \\
\hline Med16 & -2.428 & 0.018 & 0.092 & Zfp292 & -2.238 & 0.015 & 0.086 \\
\hline Med 29 & -2.799 & 0.008 & 0.064 & Zfp 30 & -6.842 & 0.002 & 0.035 \\
\hline Mfap4 & -4.773 & 0.003 & 0.045 & Zfp384 & -4.615 & 0.004 & 0.049 \\
\hline Mgat4c & -5.486 & 0.002 & 0.035 & Zfp 428 & -2.044 & 0.003 & 0.045 \\
\hline
\end{tabular}

Next, we evaluated correlation between differentially transcribed and translated mRNAs between the TG and DRG. To do this, we plotted the 379 mRNAs with higher transcript levels in TG and 315 with higher levels in the DRG. We plotted these against TPMs from the Nav1.8-TRAP datasets from both tissues. We did the same thing for the 372 Nav1.8-TRAP enriched mRNAs from TG and 348 from DRG and compared these with TPMs from input RNA sequencing (Fig. $4 C$ ). We observed that only 144 genes were shared between these datasets, suggesting that transcriptional and translational regulation is decoupled in these tissues, at least for the most highly enriched genes. This finding is consistent with genome-wide experiments showing that transcription and translation are decoupled for many, if not most, mRNAs (Liu et al., 2016).

We then sought to validate some specific findings from whole transcriptome or Nav1.8-TRAP sequencing data obtained from 

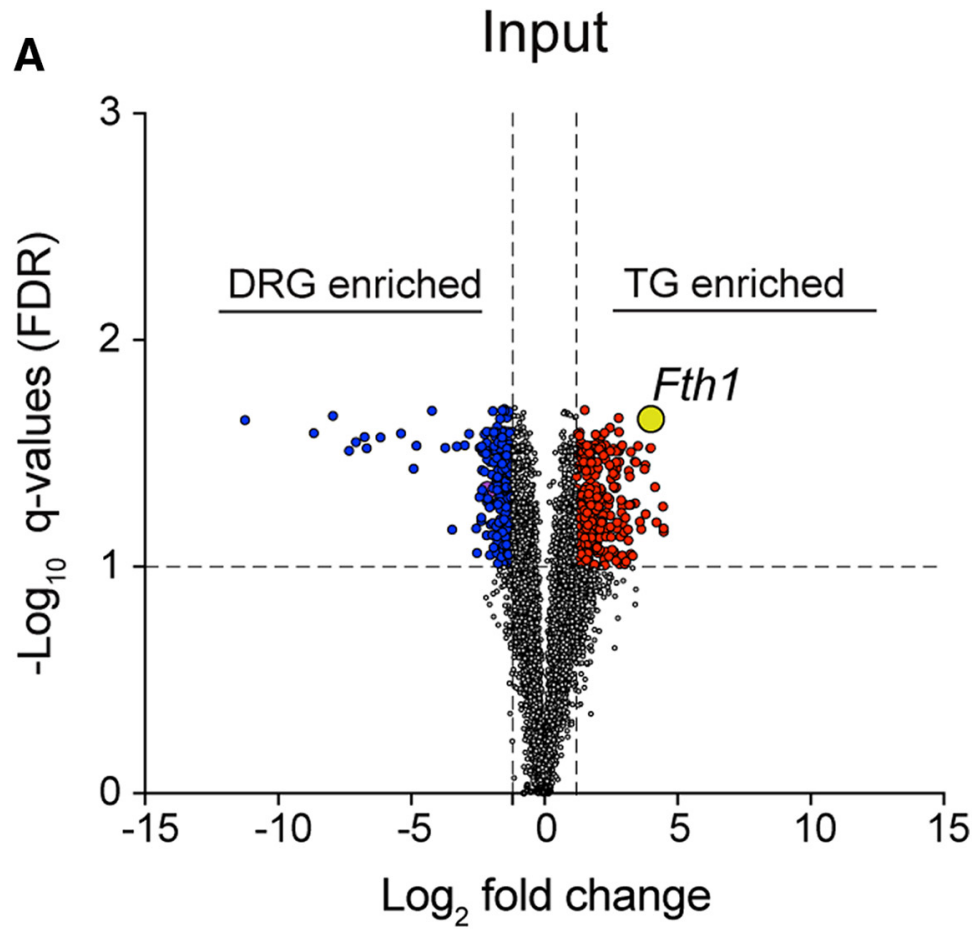

B

\section{qRT-PCR}

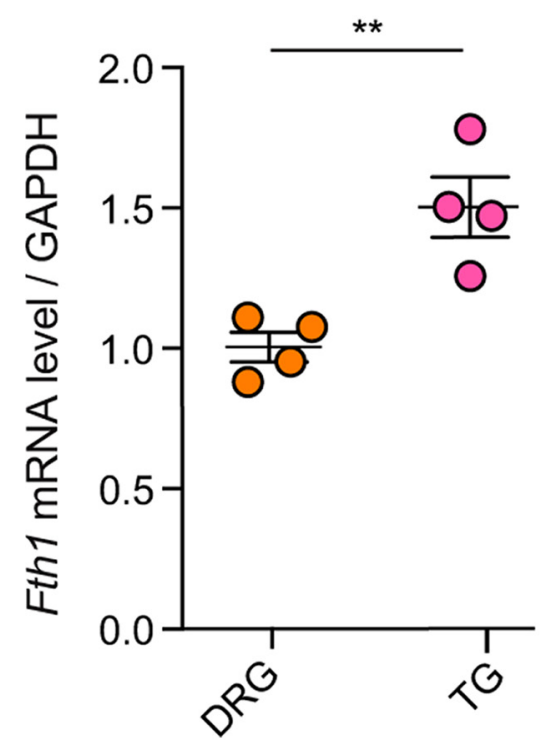

Figure 5. RNA-seq analysis reveals Fth1 as differentially expressed in the TG and validated through qRT-PCR. $\boldsymbol{A}$, Volcano plot shows Fth1 (yellow dot) as being significantly enriched in TG versus DRGs. B, qRT-PCR shows a 50\% increase in Fth1 mRNA expression in TG. (paired $t$ test, $t=4,15 ; \mathrm{df}=6 ;{ }^{* *} p=0.0048$ ).

the comparison between TG and DRG. Analysis of the differentially expressed genes between TG and DRG showed that Fth1 is highly enriched in the TG (Fig. 5A). We used qRT-PCR on mRNA prepared from both tissues to validate that there is a significant enrichment of Fth1 mRNA in the TG by this method (Fig. 5B). Comparisons of the TG and DRG transcriptome showed that multiple genes among the AMPK pathway were enriched in the DRG, such as Prkag2, Acacb, Akt1s1, and Gys (Fig. $6 A)$. Interestingly, these same mRNAs were among the 144 that were regulated at the translational level as well (Fig. $6 \mathrm{~A}$ ), but there were also a number of additional mRNAs involved in the AMPK pathway that were only found in the translatome dataset, including Cpt1c and Acaca. In stark contrast, we observed an enrichment of mRNAs in the translatome in the TG that are associated with the PI3K-mTORC1 pathway, including Strada, Lamtor5, Akt1, and Rraga (Fig. $6 A, B$ ). As mentioned previously, this predicts a higher level of mTOR activity in TG than in the DRG nociceptors. To begin to address this prediction, we examined steady-state protein levels for selected targets between DRG and TG. We chose to focus on RragA, which encodes the RagA protein, because it is a critical activator of mTORC1 activity that links mTORC1 to amino acid and glucose signaling at the interface with lysosomes (Efeyan et al., 2013, 2014). Consistent with transcriptome data, we observed no differences in the level of Rraga mRNA between TG and DRG, but we did detect a significant increase in protein level in the TG versus the DRG (Fig. 6C). We have previously shown that Rraga mRNA translation is finely controlled by the activity of Mnk1 and correlated with the level of eIF4E phosphorylation. Here, we also detected a higher level of eIF4E phosphorylation in the TG compared with DRG (Fig. 6D), suggesting that TG nociceptors may display higher translational activity via this pathway than their DRG counterparts (Megat et al., 2019). We also focused on Akt1s1, which encodes the PRAS40 protein, because this is a negative regulator of mTORC1 activity with actions that are inversely related to RagA (Wiza et al., 2012; Chong, 2016). In the DRG, we observed that the level of the ribosome-associated Akt1s1 mRNAs was higher in the DRG compared with TG, and this was validated by increased PRAS40 protein in DRG (Fig. 6E).

Collectively, the results described above suggest that the balance of mTORC1 signaling through the lysosome is shifted toward activation in the TG compared with the DRG, which could influence nociceptive responses in the facial area compared with areas innervated by the DRG. To test this hypothesis, we gave injections of a low dose of capsaicin $(0.1 \mu \mathrm{M})$, a TRPV1 agonist, into the hindpaw and the whisker pad (facial area). We observed a significantly more pronounced spontaneous pain response following facial capsaicin compared with the hindpaw (Fig. 6F). Also, the intensity/number of the nocifensive behavior was significantly larger following injection of capsaicin into the cheek, again suggesting that nociceptive stimuli trigger larger behavioral responses when administered in the facial area (Fig. $6 F$ ). We next sought to investigate whether capsaicin-induced nocifensive behavior was dependent on 
A

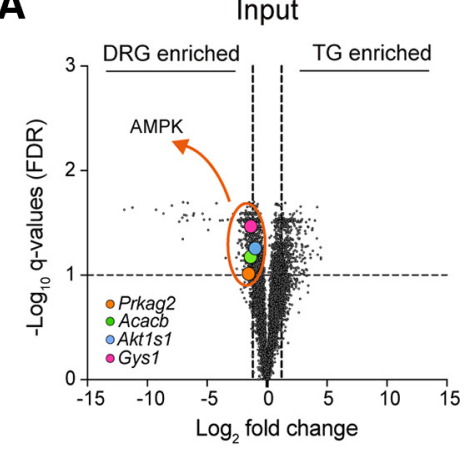

C

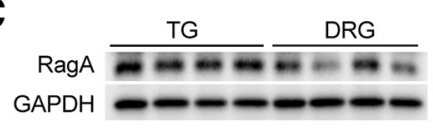

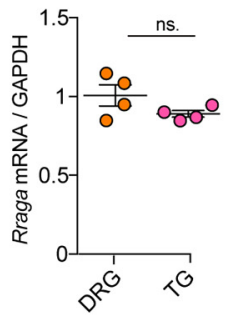

F
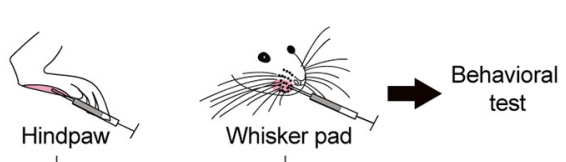

Capsaicin $(0.1 \mu \mathrm{M})$

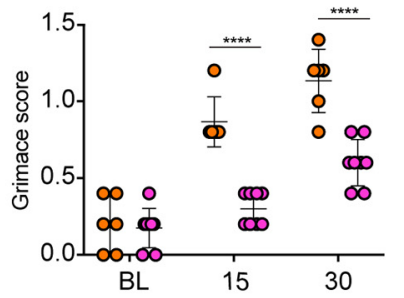

Time after capsaicin injection ( $\mathrm{min}$ )
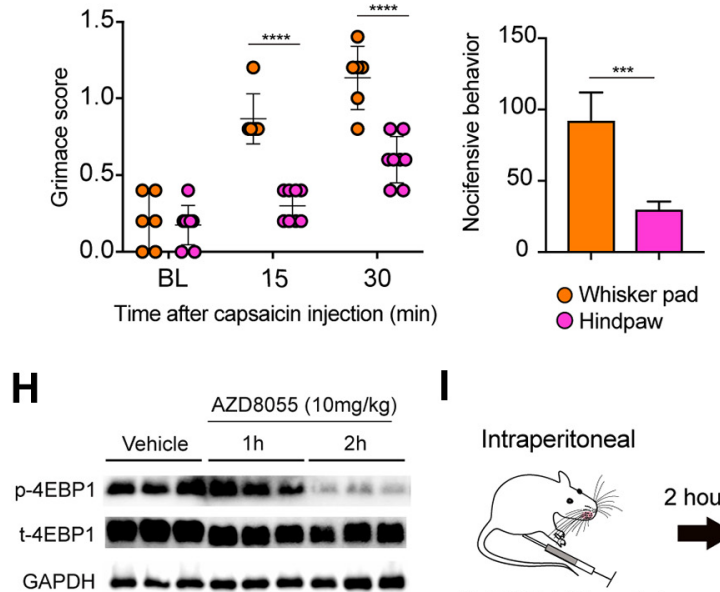

GAPDH

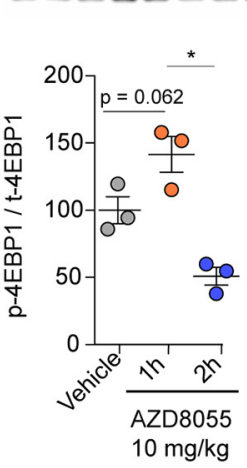

Nav1.8-TRAP

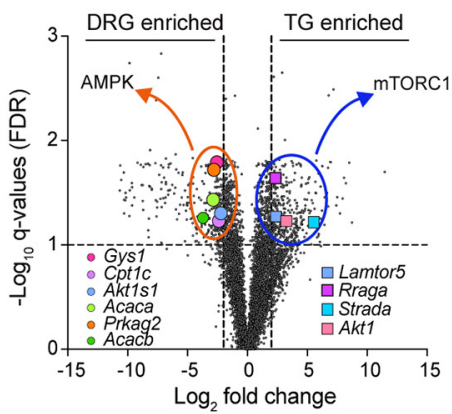

D
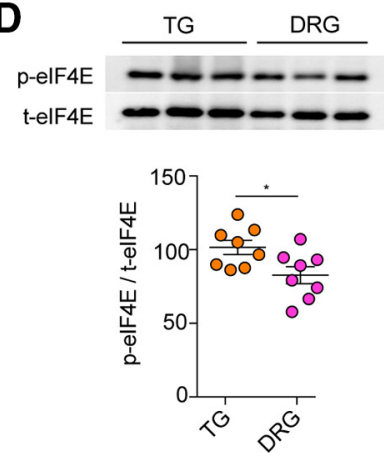

G

Intraperitoneal
B

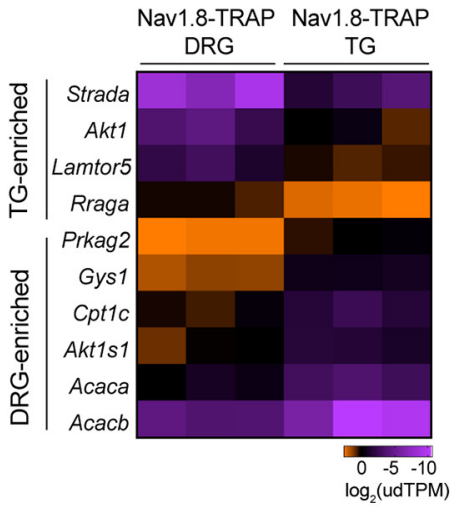

E

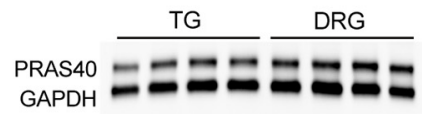

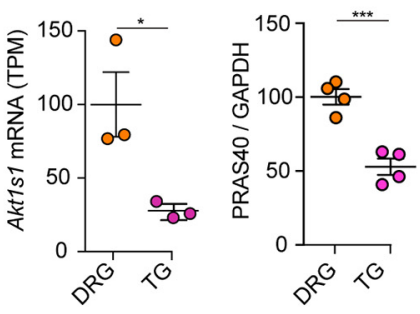

Whisker pad
Capsaicin $(0.1 \mu \mathrm{M})$
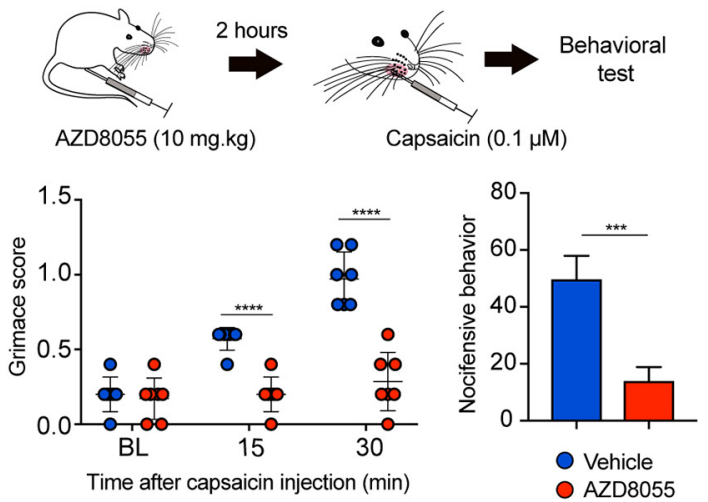

Intraperitoneal

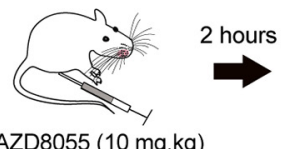

AZD8055 (10 mg.kg)

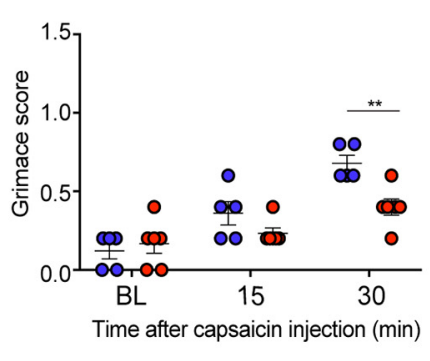

Hindpaw

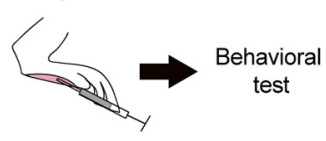

Capsaicin $(0.1 \mu \mathrm{M})$

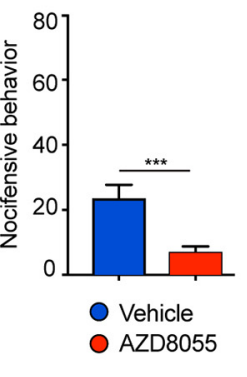

J
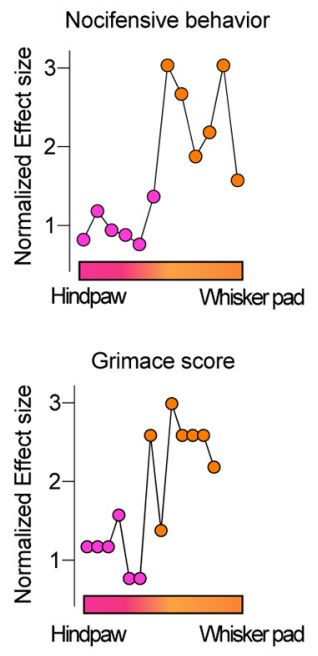

Figure 6. TRAP-seq analysis reveals that AMPK- and mTORC1-related genes are differential expressed and/or translated in the DRG and TG, respectively. $A$, Volcano plot showing an enrichment in AMPK-related genes in the input DRG sample, including Prkag2, Akt1s1, Gys1, Acacb, as well as in TRAP-seq (including Prkag2, Akt1s1, Gys1, Acacb, Acaca, and (pt1c). In converse, mTORC1-related genes are enriched in TG, such as Strada, Rraga, Akt, and Lamtor5. B, Heatmap shows increase translation of AMPK and mTORC1 genes in the DRGs and TG, respectively. (Figure legend continues.) 
mTORC1 activity in the TG region. We treated animals with an mTORC1 inhibitor (AZD8055, $10 \mathrm{mg} / \mathrm{kg}$ ) $2 \mathrm{~h}$ before injection of capsaicin into the whisker pad. We observed that the mTORC1 inhibitor significantly attenuated grimace responses and nocifensive behaviors (Fig. 6G), and this behavioral change correlated with a significant decrease in the level of p-4EBP1 (Fig. $6 \mathrm{H}$ ), a downstream target of mTORC1. While we also observed that mTORC1 inhibition significantly attenuated grimace responses and nocifensive behavior induced by a plantar injection of capsaicin (Fig. 6I), the effect size was significantly smaller compared with capsaicin into the whisker pad (Fig. 6J). Previous clinical findings reported that repetitive noxious heat stimulation, which also acts via TRPV1, creates greater sensitization in the TG region in people (Schmidt et al., 2015). Our findings parallel these observations and support a model wherein enhanced mTORC1 signaling in TG nociceptors is a cause of this enhanced sensitization.

Combining the datasets described above with single-cell RNA sequencing from existing data sources (Usoskin et al., 2015; Hu et al., 2016) allowed us to infer translation efficiencies (TEs) for all mRNAs translated in Nav1.8 neurons. First, we used the most discriminative genes in each cell type cluster (Hu et al., 2016) and calculated the correlation coefficients with all the protein coding genes in our Nav.8-TRAP sequencing datasets. Then, we plotted the heatmap of the correlation coefficient, and we observed a clear cluster of genes highly correlated with Scn10a (Fig. 7A). The Scn10a cluster (2594 genes) was compared with our TRAPfiltered dataset (7358 genes), which generated a list of 854 Scn10a-enriched genes (Fig. 7A). We then looked at the expression level of the Scn10a-enriched genes and calculated the TEs (the ratio of the TRAP and Input values) for each gene in TG and DRG datasets. Cluster 1 (C1) identified the Scn10a-enriched genes showing high TEs in the DRG (Fig. 7B; Fig. 7-1, available at https://doi.org/10.1523/JNEUROSCI.2663-18.2019.f7-1). Among them, we again found Acaca that codes for the protein ACC (acetyl-CoA carboxylase 1), a downstream target of AMPK (Har-

$\leftarrow$

(Figure legend continued.) C, Immunoblotting shows an upregulation of RagA protein inTG (RagA: DRG $\left.=100 \pm 8.39, \mathrm{~T}=149.8 \pm 8.03,{ }^{*} p=0.0003, n=11\right)$, whereas Rraga mRNA measured by qRT-PCR was not different between DRGs and TG (Rraga: DRG $=1.120 \pm 0.075$, $\mathrm{TG}=1.01 \pm 0.024, p=0.152, n=4)$. $\boldsymbol{D}$, Immunoblotting shows a lower level of elF4E phosphorylation in the DRG compared with TG (p-elF4E: TG $=100.5 \pm 4.28, \mathrm{DRG}=79.98 \pm$ $7.13,{ }^{*} p=0.0404$ ). $\boldsymbol{E}$, The negative regulator of mTORC1, PRAS40 (Akt1s1) mRNA, and TE was significantly increased in DRGs and confirmed by an increase in protein level (Akt1s1: DRG = $\left.100 \pm 5.28, \mathrm{TG}=52.62 \pm 5.48,{ }^{* * *} p=0.008, n=4\right)$. $\boldsymbol{F}$, Nocifensive behavior and grimace score after injection of capsaicin $(0.1 \mu \mathrm{m})$ into the whisker pad or the hindpaw. Capsaicin induces a more intense affective response when injected into the whisker pad compared with the hindpaw as shown by the mouse grimace score at 15 and $30 \mathrm{~min}$ (two-way ANOVA: $F_{(2,24)}=$ 22.98 , $^{* * *} p<0.0001$, post hoc Sidak ${ }^{* * * *} p<0.0001$ at 15 and 30 min). Likewise, nocifensive behavior is more pronounced when capsaicin was injected into the whisker pad compared with the hindpaw $\left(F_{(1,12)}=11.62,{ }^{* *} p<0.0052\right.$, post hoc Sidak ${ }^{* * *} p=0.002$ at 60 min after capsaicin). G, Pretreatment with an mTORC1 inhibitor (AZD8055, $10 \mathrm{mg} / \mathrm{kg}$ ) blocked capsaicininduced nocifensive behavior in the whisker pad $\left(F_{(2,24)}=13.93,{ }^{* * * *} p<0.0001\right.$, post hoc Sidak ${ }^{* *} p=0.002$ at 60 min after capsaicin) and affective pain $\left(F_{(2,24)}=21.62,{ }^{* * *} p<\right.$ 0.0001 , post hoc Sidak ${ }^{* * *} p<0.0001$ at 15 and $\left.30 \mathrm{~min}\right)$. $\boldsymbol{H}$, Intraperitoneal injection of AZD8055 $(10 \mathrm{mg} / \mathrm{kg})$ decreased the level of $\mathrm{p}-4 \mathrm{EBP} 1$ at $2 \mathrm{~h}$ (one-way ANOVA: $F_{(2,6)}=19.15$, ${ }^{* *} p=0.0025$, post hoc Dunnett: Veh vs $\left.2 \mathrm{~h},{ }^{*} p=0.027\right)$ in the TG. I, AZD8055 inhibited capsaicin-induced grimace at $30 \min \left(F_{(2,27)}=4.52,{ }^{*} p=0.02\right.$, post hoc Sidak $\left.^{* *} p=0.0034\right)$ and nocifensive behavior $\left(F_{(1,9)}=17.45,{ }^{* *} p<0.0024\right.$, post hoc Sidak ${ }^{* * *} p<0.001$ at 60 min after capsaicin) when injected into the hindpaw. J, For each group of animals, the difference between the vehicle- and AZD8055-treated values was calculated and plotted for the nocifensive behavior and mouse grimace score. We observed a significantly larger effect size of AZD8055 in nocifensive behavior (unpaired $t$ test, $t=3.52$, df $=11,{ }^{* *} p=0.0048$ ) and grimacing (unpaired $t$ test, $t=5.54, \mathrm{df}=11,{ }^{* * *} p=0.0002$ ) when capsaicin was injected in the whisker pad. ns, not significant. die, 2014). Cluster 2 (C2) identified genes showing high TEs in the TG, such as Lamtor5, Rraga, and Fkbpla, all important regulators of the mTORC1 pathway (Fig. 7B; Fig. 7-1, available at https://doi.org/10.1523/JNEUROSCI.2663-18.2019.f7-1). This cluster also identified the CGRP $\beta$ mRNA Calcb and the MrgprD receptor mRNA. Cluster (C3) contained genes with low TEs in both TG and DRG, and cluster 4 (C4) identifies genes with high TE in TG and DRG (Fig. 7-1, available at https://doi.org/10.1523/ JNEUROSCI.2663-18.2019.f7-1). Finally, we examined functional gene families (e.g., ion channels, GPCRs and kinases) for any systematic differences in TEs for mRNAs expressed in Nav1. $8^{+}$nociceptors in the TG. Interestingly, we observed that ion channels and GPCRs tend to show higher TEs compared with other gene families, such as kinases or transcription factors (Fig. 7C; Fig. 7-2, available at https://doi.org/10.1523/JNEUROSCI. 2663-18.2019.f7-2), a finding that is consistent with observations in DRG Nav1.8-expressing neurons (Megat et al., 2019).

Finally, we used MEME Suite (Bailey et al., 2015) to search for motifs in the 5' UTRs of mRNAs in clusters 1-4 described above. We only considered motifs that were found in $>30 \%$ of genes in each of the clusters. In $\mathrm{C} 1$, we did not identify any enriched motifs; however, in C2, we identified 2 motifs in mRNAs of 5' UTRs for genes with increased TE in the TG versus the DRG (Fig. 8). One of these was a GC-rich motif found in 82 of 307 mRNAs, and another was a terminal oligo pyrimidine tract motif found in 57 of 307 mRNAs. The latter motif is interesting because it is consistent with the finding that $\mathrm{mTORC} 1$ genes are more translated in the TG because terminal oligo pyrimidine tract element containing mRNAs show increased TE when mTORC1 activity is high (Thoreen et al., 2012). In the C3 cluster, which contains mRNAs with low TEs in both TG and DRG, we found a G quadruplex motif (57 of 193 mRNAs) (Fig. 8) that is likely a target for eIF4A-mediated translation control (Wolfe et al., 2014), suggesting that eIF4A activity might be low under normal conditions in TG and DRG neurons. We did not find any enriched motifs in $\mathrm{C} 4$.

\section{Discussion}

Our work uses the TRAP technology to highlight differences in the translatomes of Nav1.8 ${ }^{+}$neurons in the DRG and TG. Although there are many consistencies between these tissues, as would be expected by the similar function of Nav $1.8^{+}$neurons in the DRG and TG, there are some striking differences that may have important functional implications. Prominent among these are higher levels of protein synthesis for many regulators of the mTORC1 pathway in the TG and higher protein synthesis for members of the AMPK signaling pathway in the DRG. mTORC1 is a well-known downstream target of the AMPK kinase (Alers et al., 2012). It has been documented that, under energy-low conditions, increases in AMPK activity inhibit mTORC1, resulting in decreased overall protein synthesis and promotion of autophagy mechanisms (Schmidt et al., 2016). Because these signaling pathways regulate one another, this suggests that mRNAs that are regulated by the $\mathrm{mTORC} 1$ pathway are likely to have higher translational efficiencies in the TG than in the DRG. Previous psychophysical studies in humans have shown that painful stimulation of the TG area causes greater sensitization than stimulation of DRG-innervated regions (Schmidt et al., 2015, 2016). These studies have also demonstrated a lack of habitation in the TG region with repeated painful thermal stimulation (Schmidt et al., 2015). It is now well established that the mTORC1 signaling pathway plays a key role in controlling nociceptor excitability and sensitization (Melemedjian et al., 2010; Moy et al., 2017; Khou- 

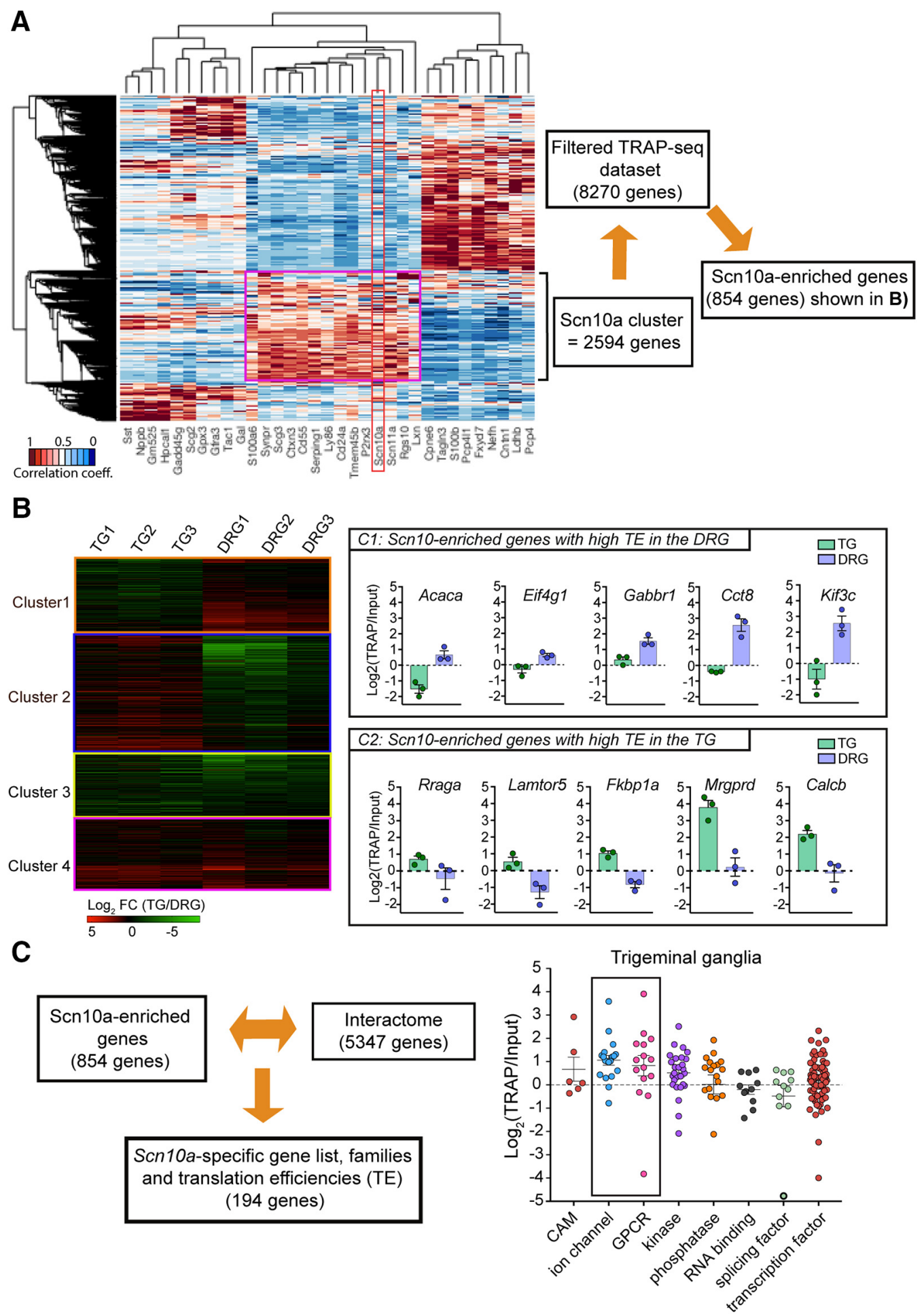

Figure 7. TE analysis for $\mathrm{Scn} 10 \mathrm{a}$-enriched genes in TG-and DRG-TRAP-seq shows differential TEs between tissues. $A$, Heatmap showing the correlation coefficient of the protein coding genes with the most discriminative expression between cell populations based on the DRG single-cell dataset published previously (Usoskin et al., 2015; Hu et al., 2016) with Nav1.8 (Scn10a) highlighted. A cluster of 2547 genes was identified as highly enriched in the Scn10a-positive neuronal population. Those 2547 genes were then merged to the TRAP-seq filtered dataset ( $\sim 8000$ genes) to identify a group of 854 mRNAs that were highly enriched in the single-cell population that also expressed Scn 10a and not found in other cell populations. B, Heatmap of the TE for the $854 \mathrm{mRNAs}$ shows 4 separate clusters. C1 identifies mRNAs with high TEs in the DRG but lower in TG. C2 shows genes with high TE in the TG and low TEs in the DRG. C3 identifies mRNAs with low TEs in both tissues. C4 identifies mRNAs with high TEs in both tissues. C, Calculation of TE efficiencies for gene families in the TG shows higher TEs for mRNAs coding for ion channels and GPCRs compared with splicing and transcription factors. Figure 7-1 (available at https://doi.org/10.1523/JNEUROSCI.2663-18.2019.f7-1) shows estimated TEs for all genes shown in clusters in Figure 7A. Figure 7-2 (available at https://doi.org/10.1523/JNEUROSCI.2663-18.2019.f7-2) shows estimated TEs by gene family.

torsky and Price, 2018), and this sensitization is strongly attenuated by activation of the AMPK pathway (Melemedjian et al., 2011; Burton et al., 2017). Our findings are in line with somatotopic differences in response to painful stimulation and a higher propensity to sensitization in TG nociceptors. While this might be explained by the biological relevance of the head and facial area for vital functions, our data show that differences in basal mTORC1 activity between TG and DRG nociceptors could drive 


\section{Motif Model}

Group

Name (e-value)

\section{Gene 5'UTRs with motif instance(s)}

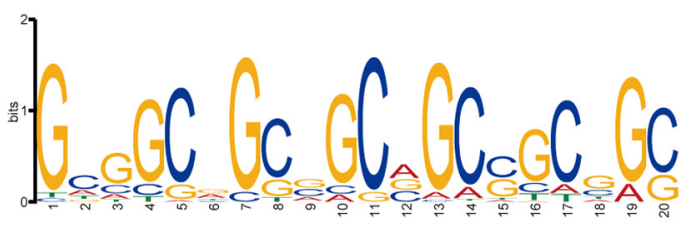

Actg1, Ankrd13b, Ankrd39, Ap1ar, Asic2, Atp6v0d1, Bag2, Basp1 Bcor, Cadm1, Car10, Ccp110, Cdyl, Col4a3bp, Coro2a, Ctdspl, Ctnna2, Disp2, Dtnbp1, Enox1, Fam124a, Fbxo9, Fkbp1a, Gabbr2, Gdpd5, Ggnbp2, Gmcl1, Gnao1, Gnaq, Gnpda1, Grm7, Hace1, Ina GC-rich motif $\quad$ Kctd15, Kif5b, Klhl5, Leprotl1, Lpar3, Lpgat1, Mapk14, Mboat7, GC-rich motif $\quad$ Kctd15, Kif5b, Klhl5, Leprotl1, Lpar3, Lpgat1, Mapk14, Mboat7, (e-score: $2.1 \times 10^{-25}$ ) Pten, Ptprt, Rab14, Rai1, Raph1, Rasgef1a, Rasgrp1, Reep5, Rer1, 82/307 genes Rexo2, Rfxap, Rnf139, Sfmbt1, Sfxn1, Sigmar1, Slc16a2, Snx13, Spag9, Srp 19, Suco, Syngr3, Tbc1d1, Tmem175, Tmem255a, Tmem86a, Trim35, Trim36, Ttc9, Ube2h, Ubl3, Ythdf2, Zdhhc2

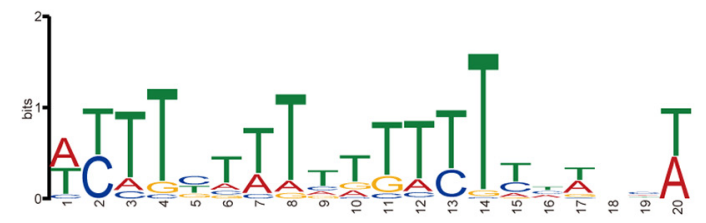

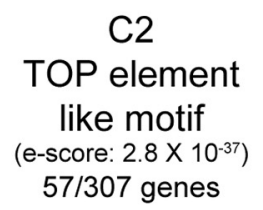

Abce1, Acs14, Adcy6, Ano3, Arl15, Atg7, Atxn2l, Car10, Cdkl2, Cebpg, Chn1, Clip4, Commd1, Cpne1, Dennd1b, Derl2, Ebf3, Elovl6, Galnt11, Galnt5, Gde1, Gnao1, Jph4, Kcnip4, Lclat1, Lgals3bp, Lrrc8b, Lrrc8d, Lrrtm2, Nrn1, Plagl1, Plexd3, Ppef1, Ppp2r5c, Ppp6r3, Pten, Rbfox1, Rho, Rnf14, Senp6, Sestd1,
Snx10, St6galnac5, Synj2bp, Synpr, Tbc1d1, Tmsb4x, Tom111, Ubald1, Ubb, Ubl3, Xiap, Zcchc18, Zdhhc13, Zeb1, Zfh×2, Zfp120

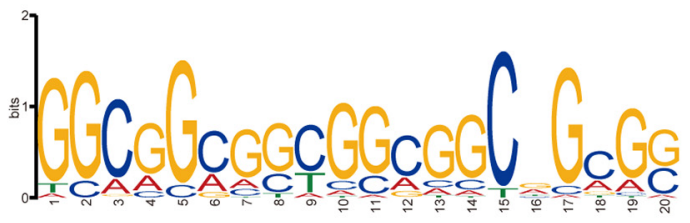

\section{C3 G-quartet like motif \\ (e-score: $\left.9.3 \times 10^{-33}\right)$ $57 / 191$ genes}

Figure 8. mRNA motifs enriched in $5^{\prime}$-UTRs from clusters of genes that show altered TEs between TG and DRG. Two motifs were found in cluster C2 (higher TE in TG than in DRG) and $1 \mathrm{motif}$ was found in cluster (3 (low TE in both TG and DRG). Genes with motifs found in their $5^{\prime}$-UTRs are shown to the right of the corresponding motifs.

differences in magnitude of sensitization following injury. However, it is also important to note that recently discovered anatomical differences between central projections of DRG and TG neurons may also mediate these differences (Rodriguez et al., 2017), in particular as they relate to enhanced fear and anxiety from painful stimulation of the TG region (Schmidt et al., 2016).

A recent paper examined differences in mRNA expression on FACS-sorted TG and DRG neurons from mice, demonstrating that $>99 \%$ of mRNA showed consistent expression between TG and DRG neurons (Lopes et al., 2017). These authors only identified 24 mRNAs with differential expression, but these included Hox genes, as we also found, and an arginine vasopressin receptor (Lopes et al., 2017). Many other differentially expressed genes they attributed to non-neuronal cell types. We found $>300$ differentially expressed genes in the whole tissue transcriptome of the DRG versus the TG, and many of these mRNAs may be attributable to non-neuronal cells because we did not sort cells for whole transcriptome. This likely explains the major discrepancies between transcriptomes in these two papers. However, major differences in translatome findings cannot be attributable to nonneuronal cells because the approach we use is specific to Nav1.8expressing neurons, most of which are nociceptors. Our work also identifies potential differences in translation regulation signaling between the DRG and TG that provides a plausible explanation for these difference in the translatome. This is especially important considering that the mTOR (Patursky-Polischuk et al., 2009; Thoreen et al., 2012) and AMPK (Dowling et al., 2007) pathways have distinct effects on TE of specific subsets of mRNAs.

There are limitations to our approach. Primary among these is that our TE estimates could only be applied to a subset of genes that have been identified as highly enriched in the Nav1.8 ${ }^{+}$population of neurons by single-cell RNA sequencing. Future efforts may use cell-sorting techniques (Thakur et al., 2014; Lopes et al., 2017) for transcriptome generation in combination with TRAP sequencing to make estimates of the TE across the active genome of Nav1.8 $8^{+}$population of cells. A technical shortcoming of this potential approach is that tissue homogenization and cellular dissociation protocols that are needed to sort cells for transcriptomic analysis cause induction of classes of genes, including molecular chaperones and immediate early genes that can bias transcriptomes and distort TE estimates (van den Brink et al., 2017). A second limitation is that, while our data are suggestive of important differences in $\mathrm{mTORC} 1$ and AMPK signaling between these two tissues that may regulate susceptibility to nociceptor sensitization, we have not shown this directly with behavioral or electrophysiological evidence. However, the notion of that TG nociceptors are more intensely sensitized by noxious stimuli is supported by preclinical models and human psychophysical data (Schmidt et al., 2015, 2016). For example, it has recently been demonstrated that injury to TG nerves induces a grimacing effect in both rats and mice (Akintola et al., 2017). This is in stark contrast to effects of injury to the sciatic nerve where grimacing effects are not observed (Langford et al., 2010). These findings suggest that injury to TG nerves induces a stronger ongoing pain phenotype in both of these rodent species. Additional work is needed to clarify whether this is driven by the mTOR signaling axis in the TG.

The results presented here add to a growing body of literature that there are important differences between the DRG and TG that are likely relevant for understanding pain disorders that originate from these regions. These include differential developmen- 
tal origins (Zou et al., 2004), differential expression of neuronal subtype markers (Price and Flores, 2007), and altered response to injury, such as sympathetic sprouting into the DRG in response to injury (Chung et al., 1996; Chien et al., 2005; Xie et al., 2007, 2015), which does not occur in the TG (Bongenhielm et al., 1999). Our use of the TRAP technique to define the translatomes of Nav1. $8^{+}$neurons in DRG and TG points to a host of newly discovered differences between these two tissues and generates a new resource that can be mined to gain addition insight.

\section{References}

Akintola T, Raver C, Studlack P, Uddin O, Masri R, Keller A (2017) The grimace scale reliably assesses chronic pain in a rodent model of trigeminal neuropathic pain. Neurobiol Pain 2:13-17.

Alers S, Loffler AS, Wesselborg S, Stork B (2012) Role of AMPK-mTORUlk1/2 in the regulation of autophagy: cross talk, shortcuts, and feedbacks. Mol Cell Biol 32:2-11.

Bailey TL, Johnson J, Grant CE, Noble WS (2015) The MEME Suite. Nucleic Acids Res 43:W39-W49.

Bongenhielm U, Boissonade FM, Westermark A, Robinson PP, Fried K (1999) Sympathetic nerve sprouting fails to occur in the trigeminal ganglion after peripheral nerve injury in the rat. Pain 82:283-288.

Burton MD, Tillu DV, Mazhar K, Mejia GL, Asiedu MN, Inyang K, Hughes T, Lian B, Dussor G, Price TJ (2017) Pharmacological activation of AMPK inhibits incision-evoked mechanical hypersensitivity and the development of hyperalgesic priming in mice. Neuroscience 359:119-129.

Chien SQ, Li C, Li H, Xie W, Pablo CS, Zhang JM (2005) Sympathetic fiber sprouting in chronically compressed dorsal root ganglia without peripheral axotomy. J Neuropathic Pain Symptom Palliation 1:19-23.

Chong ZZ (2016) Targeting PRAS40 for multiple diseases. Drug Discov Today 21:1222-1231.

Chung K, Lee BH, Yoon YW, Chung JM (1996) Sympathetic sprouting in the dorsal root ganglia of the injured peripheral nerve in a rat neuropathic pain model. J Comp Neurol 376:241-252.

Devor M (1999) Unexplained peculiarities of the dorsal root ganglion. Pain Suppl 6:S27-S35.

Dowling RJ, Zakikhani M, Fantus IG, Pollak M, Sonenberg N (2007) Metformin inhibits mammalian target of rapamycin-dependent translation initiation in breast cancer cells. Cancer Res 67:10804-10812.

Doyle JP, Dougherty JD, Heiman M, Schmidt EF, Stevens TR, Ma G, Bupp S, Shrestha P, Shah RD, Doughty ML, Gong S, Greengard P, Heintz N (2008) Application of a translational profiling approach for the comparative analysis of CNS cell types. Cell 135:749-762.

Dubin AE, Patapoutian A (2010) Nociceptors: the sensors of the pain pathway. J Clin Invest 120:3760-3772.

Durham PL, Garrett FG (2010) Development of functional units within trigeminal ganglia correlates with increased expression of proteins involved in neuron-glia interactions. Neuron Glia Biol 6:171-181.

Efeyan A, Zoncu R, Chang S, Gumper I, Snitkin H, Wolfson RL, Kirak O, Sabatini DD, Sabatini DM (2013) Regulation of mTORC1 by the rag GTPases is necessary for neonatal autophagy and survival. Nature 493: $679-683$.

Efeyan A, Schweitzer LD, Bilate AM, Chang S, Kirak O, Lamming DW, Sabatini DM (2014) RagA, but not RagB, is essential for embryonic development and adult mice. Dev Cell 29:321-329.

Flegel C, Schöbel N, Altmüller J, Becker C, Tannapfel A, Hatt H, Gisselmann G (2015) RNA-Seq analysis of human trigeminal and dorsal root ganglia with a focus on chemoreceptors. PLoS One 10:e0128951.

Fortelny N, Overall CM, Pavlidis P, Freue GV (2017) Can we predict protein from mRNA levels? Nature 547:E19-E20.

Glusman G, Caballero J, Robinson M, Kutlu B, Hood L (2013) Optimal scaling of digital transcriptomes. PLoS One 8:e77885.

Gong L, Wu J, Zhou S, Wang Y, Qin J, Yu B, Gu X, Yao C (2016) Global analysis of transcriptome in dorsal root ganglia following peripheral nerve injury in rats. Biochem Biophys Res Commun 478:206-212.

Hardie DG (2014) AMPK-sensing energy while talking to other signaling pathways. Cell Metab 20:939-952.

Hardie DG (2015) AMPK: positive and negative regulation, and its role in whole-body energy homeostasis. Curr Opin Cell Biol 33:1-7.

Heiman M, Schaefer A, Gong S, Peterson JD, Day M, Ramsey KE, SuárezFariñas M, Schwarz C, Stephan DA, Surmeier DJ, Greengard P, Heintz N
(2008) A translational profiling approach for the molecular characterization of CNS cell types. Cell 135:738-748.

Heiman M, Kulicke R, Fenster RJ, Greengard P, Heintz N (2014) Cell typespecific mRNA purification by translating ribosome affinity purification (TRAP). Nat Protoc 9:1282-1291.

Hu G, Huang K, Hu Y, Du G, Xue Z, Zhu X, Fan G (2016) Single-cell RNA-seq reveals distinct injury responses in different types of DRG sensory neurons. Sci Rep 6:31851.

Ingolia NT (2016) Ribosome footprint profiling of translation throughout the genome. Cell 165:22-33.

Isensee J, Wenzel C, Buschow R, Weissmann R, Kuss AW, Hucho T (2014) Subgroup-elimination transcriptomics identifies signaling proteins that define subclasses of TRPV1-positive neurons and a novel paracrine circuit. PLoS One 9:e115731.

Kammermeier L, Reichert H (2001) Common developmental genetic mechanisms for patterning invertebrate and vertebrate brains. Brain Res Bull 55:675-682.

Khoutorsky A, Price TJ (2018) Translational control mechanisms in persistent pain. Trends Neurosci 41:100-114.

Kogelman LJ, Christensen RE, Pedersen SH, Bertalan M, Hansen TF, JansenOlesen I, Olesen J (2017) Whole transcriptome expression of trigeminal ganglia compared with dorsal root ganglia in rattus norvegicus. Neuroscience 350:169-179.

Kuleshov MV, Jones MR, Rouillard AD, Fernandez NF, Duan Q, Wang Z, Koplev S, Jenkins SL, Jagodnik KM, Lachmann A, McDermott MG, Monteiro CD, Gundersen GW, Ma'ayan A (2016) Enrichr: a comprehensive gene set enrichment analysis web server 2016 update. Nucleic Acids Res 44:W90-W97.

Langford DJ, Bailey AL, Chanda ML, Clarke SE, Drummond TE, Echols S, Glick S, Ingrao J, Klassen-Ross T, Lacroix-Fralish ML, Matsumiya L, Sorge RE, Sotocinal SG, Tabaka JM, Wong D, van den Maagdenberg AM, Ferrari MD, Craig KD, Mogil JS (2010) Coding of facial expressions of pain in the laboratory mouse. Nat Methods 7:447-449.

Liu Y, Beyer A, Aebersold R (2016) On the dependency of cellular protein levels on mRNA abundance. Cell 165:535-550.

Lopes DM, Denk F, McMahon SB (2017) The molecular fingerprint of dorsal root and trigeminal ganglion neurons. Front Mol Neurosci 10:304.

Manteniotis S, Lehmann R, Flegel C, Vogel F, Hofreuter A, Schreiner BS, Altmüller J, Becker C, Schöbel N, Hatt H, Gisselmann G (2013) Comprehensive RNA-seq expression analysis of sensory ganglia with a focus on ion channels and GPCRs in trigeminal ganglia. PLoS One 8:e79523.

Megat S, Ray PR, Moy JK, Lou TF, Barragán-Iglesias P, Li Y, Pradhan G, Wanghzou A, Ahmad A, Burton MD, North RY, Dougherty PM, Khoutorsky A, Sonenberg N, Webster KR, Dussor G, Campbell ZT, Price TJ (2019) Nociceptor translational profiling reveals the RagA-mTORC1 network as a critical generator of neuropathic pain. J Neurosci 39:393411.

Melemedjian OK, Asiedu MN,Tillu DV, Sanoja R, Yan J, Lark A, Khoutorsky A, Johnson J, Peebles KA, Lepow T, Sonenberg N, Dussor G, Price TJ (2011) Targeting adenosine monophosphate-activated protein kinase (AMPK) in preclinical models reveals a potential mechanism for the treatment of neuropathic pain. Mol Pain 7:70.

Melemedjian OK, Asiedu MN, Tillu DV, Peebles KA, Yan J, Ertz N, Dussor GO, Price TJ (2010) IL-6- and NGF-induced rapid control of protein synthesis and nociceptive plasticity via convergent signaling to the eIF4F complex. J Neurosci 30:15113-15123.

Moy JK, Khoutorsky A, Asiedu MN, Black BJ, Kuhn JL, Barragán-Iglesias P, Megat S, Burton MD, Burgos-Vega CC, Melemedjian OK, Boitano S, Vagner J, Gkogkas CG, Pancrazio JJ, Mogil JS, Dussor G, Sonenberg N, Price TJ (2017) The MNK-eIF4E signaling axis contributes to injuryinduced nociceptive plasticity and the development of chronic pain. J Neurosci 37:7481-7499.

Patursky-Polischuk I, Stolovich-Rain M, Hausner-Hanochi M, Kasir J, Cybulski N, Avruch J, Rüegg MA, Hall MN, Meyuhas O (2009) The TSCmTOR pathway mediates translational activation of TOP mRNAs by insulin largely in a raptor- or rictor-independent manner. Mol Cell Biol 29:640-649.

Price TJ, Flores CM (2007) Critical evaluation of the colocalization between calcitonin gene-related peptide, substance $\mathrm{P}$, transient receptor potential vanilloid subfamily type 1 immunoreactivities, and isolectin B4 binding in primary afferent neurons of the rat and mouse. J Pain 8:263-272.

Reynders A, Mantilleri A, Malapert P, Rialle S, Nidelet S, Laffray S, Beurrier C, 
Bourinet E, Moqrich A (2015) Transcriptional profiling of cutaneous MRGPRD free nerve endings and C-LTMRs. Cell Rep 10:1007-1019.

Rodriguez E, Sakurai K, Xu J, Chen Y, Toda K, Zhao S, Han BX, Ryu D, Yin H, Liedtke W, Wang F (2017) A craniofacial-specific monosynaptic circuit enables heightened affective pain. Nat Neurosci 20:1734-1743.

Schmidt K, Schunke O, Forkmann K, Bingel U (2015) Enhanced short-term sensitization of facial compared with limb heat pain. J Pain 16:781-790.

Schmidt K, Forkmann K, Sinke C, Gratz M, Bitz A, Bingel U (2016) The differential effect of trigeminal vs. peripheral pain stimulation on visual processing and memory encoding is influenced by pain-related fear. Neuroimage 134:386-395.

Stirling LC, Forlani G, Baker MD, Wood JN, Matthews EA, Dickenson AH, Nassar MA (2005) Nociceptor-specific gene deletion using heterozygous NaV1.8-cre recombinase mice. Pain 113:27-36.

Takeda M, Takahashi M, Matsumoto S (2008) Contribution of activated interleukin receptors in trigeminal ganglion neurons to hyperalgesia via satellite glial interleukin-1beta paracrine mechanism. Brain Behav Immun 22:1016-1023.

Thakur M, Crow M, Richards N, Davey GI, Levine E, Kelleher JH, Agley CC, Denk F, Harridge SD, McMahon SB (2014) Defining the nociceptor transcriptome. Front Mol Neurosci 7:87.

Thoreen CC, Chantranupong L, Keys HR, Wang T, Gray NS, Sabatini DM (2012) A unifying model for mTORC1-mediated regulation of mRNA translation. Nature 485:109-113.

Usoskin D, Furlan A, Islam S, Abdo H, Lönnerberg P, Lou D, Hjerling-Leffler J, Haeggström J, Kharchenko O, Kharchenko PV, Linnarsson S, Ernfors P (2015) Unbiased classification of sensory neuron types by large-scale single-cell RNA sequencing. Nat Neurosci 18:145-153.

Uttam S, Wong C, Amorim IS, Jafarnejad SM, Tansley SN, Yang J, PragerKhoutorsky M, Mogil JS, Gkogkas CG, Khoutorsky A (2018) Transla- tional profiling of dorsal root ganglia and spinal cord in a mouse model of neuropathic pain. Neurobiol Pain 4:35-44.

van den Brink SC, Sage F, Vértesy Á, Spanjaard B, Peterson-Maduro J, Baron CS, Robin C, van Oudenaarden A (2017) Single-cell sequencing reveals dissociation-induced gene expression in tissue subpopulations. Nat Methods 14:935-936.

Wiza C, Nascimento EB, Ouwens DM (2012) Role of PRAS40 in Akt and mTOR signaling in health and disease. Am J Physiol Endocrinol Metab 302:E1453-E1460.

Wolfe AL, Singh K, Zhong Y, Drewe P, Rajasekhar VK, Sanghvi VR, Mavrakis KJ, Jiang M, Roderick JE, Van der Meulen J, Schatz JH, Rodrigo CM, Zhao C, Rondou P, de Stanchina E, Teruya-Feldstein J, Kelliher MA, Speleman F, Porco JA Jr, Pelletier J, et al. (2014) RNA G-quadruplexes cause eIF4A-dependent oncogene translation in cancer. Nature 513:65-70.

Woolf CJ, Ma Q (2007) Nociceptors-noxious stimulus detectors. Neuron 55:353-364.

Xie W, Strong JA, Li H, Zhang JM (2007) Sympathetic sprouting near sensory neurons after nerve injury occurs preferentially on spontaneously active cells and is reduced by early nerve block. J Neurophysiol 97:492-502.

Xie W, Strong JA, Zhang JM (2015) Local knockdown of the NaV1.6 sodium channel reduces pain behaviors, sensory neuron excitability, and sympathetic sprouting in rat models of neuropathic pain. Neuroscience 291:317-330

Zhou P, Zhang Y, Ma Q, Gu F, Day DS, He A, Zhou B, Li J, Stevens SM, Romo $\mathrm{D}, \mathrm{Pu}$ WT (2013) Interrogating translational efficiency and lineagespecific transcriptomes using ribosome affinity purification. Proc Natl Acad Sci U S A 110:15395-15400.

Zou D, Silvius D, Fritzsch B, Xu PX (2004) Eyal and Six1 are essential for early steps of sensory neurogenesis in mammalian cranial placodes. Development 131:5561-5572. 